\title{
Inclusive Search for a Highly Boosted Higgs Boson Decaying to a Bottom Quark-Antiquark Pair
}

\author{
A. M. Sirunyan et al. \\ (CMS Collaboration)
}

(Received 16 September 2017; published 14 February 2018)

\begin{abstract}
An inclusive search for the standard model Higgs boson $(H)$ produced with large transverse momentum $\left(p_{T}\right)$ and decaying to a bottom quark-antiquark pair $(b \bar{b})$ is performed using a data set of $p p$ collisions at $\sqrt{s}=13 \mathrm{TeV}$ collected with the CMS experiment at the LHC. The data sample corresponds to an integrated luminosity of $35.9 \mathrm{fb}^{-1}$. A highly Lorentz-boosted Higgs boson decaying to $b \bar{b}$ is reconstructed as a single, large radius jet, and it is identified using jet substructure and dedicated $b$ tagging techniques. The method is validated with $Z \rightarrow b \bar{b}$ decays. The $Z \rightarrow b \bar{b}$ process is observed for the first time in the single-jet topology with a local significance of 5.1 standard deviations (5.8 expected). For a Higgs boson mass of $125 \mathrm{GeV}$, an excess of events above the expected background is observed (expected) with a local significance of 1.5 (0.7) standard deviations. The measured cross section times branching fraction for production via gluon fusion of $H \rightarrow b \bar{b}$ with reconstructed $p_{T}>450 \mathrm{GeV}$ and in the pseudorapidity range $-2.5<\eta<2.5$ is $74 \pm 48$ (stat) ${ }_{-10}^{+17}$ (syst) $\mathrm{fb}$, which is consistent within uncertainties with the standard model prediction.
\end{abstract}

DOI: 10.1103/PhysRevLett.120.071802

In the standard model (SM) [1-3], the Brout-EnglertHiggs mechanism [4-8] is responsible for electroweak symmetry breaking and the mass of elementary particles. Although a Higgs boson $(H)$ was discovered [9-11], the LHC data sets of $p p$ collisions at $\sqrt{s}=7$ and $8 \mathrm{TeV}$ were not sufficient to establish the coupling to bottom quarks [12], despite the $58.1 \%$ expected branching fraction of the Higgs boson to bottom quark-antiquark $(b \bar{b})$ pairs [13]. The most sensitive method to search for $H \rightarrow b \bar{b}$ decays at a hadron collider is to use events in which the Higgs boson is produced in association with a $W$ or $Z$ boson $(V H)$ decaying to leptons, and recoiling with a large transverse momentum $\left(p_{T}\right)$ [14], in order to suppress the overwhelming irreducible background from quantum chromodynamics (QCD) multijet production of $b$ quarks. Because of this background, an observation of $H(b \bar{b})$ decays in the gluon fusion production mode (GGF) as considered impossible. This Letter presents the first inclusive search for $H \rightarrow b \bar{b}$, where the Higgs boson is produced with high- $p_{T}$. Measurements of high- $p_{T} H(b \bar{b})$ decays may resolve the loop induced and tree-level contributions to the GGF process [15] and provide an alternative approach to study

\footnotetext{
*Full author list given at the end of the article.

Published by the American Physical Society under the terms of the Creative Commons Attribution 4.0 International license. Further distribution of this work must maintain attribution to the author(s) and the published article's title, journal citation, and DOI. Funded by SCOAP ${ }^{3}$.
}

the top quark Yukawa coupling in addition to the $t \bar{t} H$ process.

The results reported in this Letter are based on a data set of $p p$ collisions at $\sqrt{s}=13 \mathrm{TeV}$, collected with the CMS detector at the LHC in 2016, and corresponding to an integrated luminosity of $35.9 \mathrm{fb}^{-1}$. The main experimental difficulties for this search originate from the large cross section for background multijet events at low jet mass and the restrictive trigger requirements needed to reduce the data recording rate. Therefore, we require events to have a high- $p_{T}$ Higgs boson candidate and define six $p_{T}$ categories from $450 \mathrm{GeV}$ to $1 \mathrm{TeV}$ with variable width from 50 to $200 \mathrm{GeV}$. Combinatorial backgrounds are reduced by requiring the Higgs boson's decay products to be clustered in a single jet [14]. The jet is required to have a two-prong substructure and $b$ tagging properties consistent with the $H(b \bar{b})$ signal. The nontrivial jet mass shape is difficult to model parametrically. For this reason, the dominant background from SM QCD multijet production is estimated in data by inverting the $b$ tagging requirement, which is, by design, decorrelated from jet mass and $p_{T}$. A simultaneous fit to the distributions of the jet mass in all categories is performed in the range 40 to $201 \mathrm{GeV}$ to extract the inclusive $H(b \bar{b})$ and $Z(b \bar{b})$ production cross sections and to determine the normalizations and shapes of the jet mass distributions for the backgrounds.

A detailed description of the CMS detector, together with a definition of the coordinate system and the relevant kinematic variables, can be found in Ref. [16]. The central feature of the CMS apparatus is a superconducting solenoid 
of $6 \mathrm{~m}$ internal diameter, providing a magnetic field of 3.8 T. A silicon pixel and strip tracker, a lead tungstate crystal electromagnetic calorimeter, and a brass and scintillator hadron calorimeter, each composed of a barrel and two end cap sections, reside within the solenoid. Forward calorimeters extend the pseudorapidity $(\eta)[16]$ coverage provided by the barrel and end cap detectors. Muons are detected in gas-ionization chambers embedded in the steel flux-return yoke outside the solenoid.

Simulated samples of signal and background events are produced using various Monte Carlo (MC) event generators, with the CMS detector response modeled using the GEANT4 [17] program. The MADGRAPH5_aMC@NLO 2.3.3 [18] generator is used for the diboson, $W+$ jets, $Z+$ jets, QCD multijet samples at leading order (LO) accuracy, with matching [19] between jets from the matrix element calculation and the parton shower description, while POWHEG 2.0 [20-22] at next-to-leading order (NLO) precision is used to model the $t \bar{t}$ and single-top processes. For parton showering and hadronization, the POWHEG and MADGRAPH5_aMC@NLO samples are interfaced with PYTHIA 8.212 [23]. The PYTHIA parameters for the underlying event description are set to the CUETP8M1 tune [24]. The production cross sections for the diboson samples are calculated to next-to-next-to-leading-order (NNLO) accuracy with the MCFM 7.0 program [25]. The cross section for top quark pair production is computed with ToP++ 2.0 [26] at NNLO. The cross sections for $W+$ jets and $Z+$ jets samples include higher-order QCD and electroweak (EW) corrections and improve modeling of high- $p_{T} W$ and $Z$ bosons events [27-30]. The parton distribution function (PDF) set NNPDF3.0 [31] is used to produce all simulated samples, with the accuracy (LO or NLO) corresponding to that of the generator used. The Higgs boson signal samples are produced using the POWHEG event generator with $m_{H}=125 \mathrm{GeV}$. For the GGF production mode, the POWHEG generated sample with up to one extra jet in matrix element calculations is normalized to the inclusive cross section at next-to-next-to-next-to-leading order $\left(\mathrm{N}^{3} \mathrm{LO}\right)$ accuracy [32-35]. The resulting Higgs boson $p_{T}$ spectrum neglects the effects of the finite top quark mass [36] and associated higher-order QCD corrections [37-40], which are expected to be large for $p_{T}$ greater than approximately twice the mass of the top quark [36]. A $p_{T}$-dependent correction has been derived to account for both of these effects. The POWHEG GGF $p_{T}$ spectrum is reweighted to the $0-2$ jet CKKW-L $[26,41,42]$ merged LO GGF process incorporating the finite top quark mass $\left(m_{t}\right)$ [13,43-45]. This spectrum is then corrected by the approximate NLO to LO ratio, obtained by expanding in powers of $1 / m_{t}^{2}$ up to $1 / m_{t}^{4}$, and the effective NNLO to NLO ratio $[46,47]$ in the infinite top quark mass approximation. The overall $p_{T}$-dependent correction to the initial $\mathrm{N}^{3} \mathrm{LO}$ POWHEG GGF spectrum is found to be $1.27 \pm 0.38$, resulting in a GGF cross section times $H(b \bar{b})$ branching fraction of $31.7 \pm 9.5 \mathrm{fb}$ for reconstructed Higgs boson $p_{T}>450 \mathrm{GeV}$ and $|\eta|<2.5$. An uncertainty of $30 \%$ to the overall correction is estimated from the comparison of different predictions obtained by using (i) a merging scale of 100 instead of $20 \mathrm{GeV}$, (ii) the inclusive two-jet GGF process generation, and (iii) the MADGRAPH5_aMC@NLO effective field theory approximation $[13,46]$ normalized to the inclusive $\mathrm{N}^{3} \mathrm{LO}$ cross section. The $p_{T}$ spectrum of the Higgs boson for the vector boson fusion $(\mathrm{VBF})$ production mode is reweighted to account for $\mathrm{N}^{3} \mathrm{LO}$ corrections to the cross section. These corrections $[48,49]$ have a negligible effect on the yield for this process for events with Higgs boson $p_{T}>450 \mathrm{GeV}$.

The particle-flow event algorithm [50] is employed to reconstruct and identify each individual particle with an optimized combination of information from the various elements of the CMS detector. The algorithm identifies each reconstructed particle as an electron, a muon, a photon, or a charged or a neutral hadron. The missing transverse momentum vector is defined as the negative vectorial sum of the transverse momenta of all the particles identified in the event, and its magnitude is referred to as $p_{T}^{\text {miss }}$.

The particles are clustered into jets using the anti- $k_{T}$ algorithm [51] with a distance parameter of 0.8 (AK8 jets). To mitigate the effect of pileup, the pileup per particle identification (PUPPI) algorithm [52] assigns a weight to each particle prior to jet clustering based on the likelihood of the particle originating from the hard scattering vertex. Further corrections are applied to the jet energy as a function of jet $\eta$ and $p_{T}$ to account for detector response nonlinearities.

To isolate the Higgs boson signal, a high- $p_{T}$ signal jet is required. Combinations of several online selections are used, all requiring the total hadronic transverse energy in the event $\left(H_{T}\right)$ or jet $p_{T}$ to be above a given threshold. In addition, a minimum threshold on the jet mass is imposed after removing remnants of soft radiation with the jet trimming technique [53] to reduce the $H_{T}$ or $p_{T}$ thresholds and improve the signal acceptance. The online selection is fully efficient at selecting events offline with at least one AK8 jet with $p_{T}>450 \mathrm{GeV}$ and $|\eta|<2.5$. Events containing identified and isolated electrons, muons, or $\tau$ leptons with $p_{T}>10,10$, or $18 \mathrm{GeV}$ and $|\eta|<2.5,2.4$, or 2.3, respectively, are vetoed to reduce backgrounds from SM EW processes. Since no genuine $p_{T}^{\text {miss }}$ is expected for signal processes, events with $p_{T}^{\text {miss }}>140 \mathrm{GeV}$ are removed in order to further reduce the top quark background contamination. The leading (in $p_{T}$ ) jet in the event is assumed to be the Higgs boson candidate, the $H$ jet. The soft-drop algorithm [54,55] is used to remove soft and wide-angle radiation with a soft radiation fraction $z$ less than 0.1 . The parameter $\beta$ is set to zero, which corresponds to the case in which approximately the same fraction of energy is groomed away, regardless of the initial jet energy. 
The use of soft-drop grooming reduces the jet mass $\left(m_{\mathrm{SD}}\right)$ for background QCD events when large jet masses arise from soft gluon radiation. For signal events, the jet mass is primarily determined by the $H(b \bar{b})$ decay kinematics and its distribution peaks at the mass of the Higgs boson. Dedicated $m_{\mathrm{SD}}$ corrections [56] are derived from simulation and data in a region enriched with merged $W(q \bar{q})$ decays from $t \bar{t}$ events. They remove a residual dependence on the jet $p_{T}$ and match the jet mass scale and resolution to those observed in data.

The dimensionless mass scale variable for $\mathrm{QCD}$ jets, $\rho=$ $\log \left(m_{\mathrm{SD}}{ }^{2} / p_{T}^{2}\right)$ [54,57], whose distribution is roughly invariant in different ranges of jet $p_{T}$, is used to characterize the correlation between the jet $b$ tagging discriminator, jet mass, and jet $p_{T}$. Only events in the range $-6.0<\rho<$ -2.1 are considered, to avoid instabilities at the edges of the distribution due to finite cone limitations from the AK8 jet clustering $(\rho \gtrsim-2.1)$ and to avoid the nonperturbative regime of the soft-drop mass calculation $(\rho \lesssim-6.0)$. This requirement is fully efficient for the Higgs boson signal.

The $N_{2}^{1}$ variable [58], which is based on a ratio of 2-point and 3-point generalized energy correlation functions (ECFs) [59], is exploited to determine how consistent a jet is with having a two-prong substructure. The calculation of $N_{2}^{1}$ is based on the jet constituents after application of the soft-drop grooming algorithm to the jet. It provides excellent discrimination between two-prong signal jets and QCD background jets [58]. However, any selection on $N_{2}^{1}$ or other similar variables [60] shapes the jet mass distributions differently depending on the $p_{T}$ of the jet. Therefore a transformation of $N_{2}^{1}$ to $N_{2}^{1, D D T}$ is applied, where DDT stands for designed decorrelated tagger [57], to reduce its correlation with $\rho$ and $p_{T}$ in multijet events. We define $N_{2}^{1, \text { DDT }}=N_{2}^{1}-N_{2(26 \%)}^{1}$, where $N_{2(26 \%)}^{1}$ is the 26th percentile of the $N_{2}^{1}$ distribution in simulated QCD events as a function of $\rho$ and $p_{T}$. This ensures that the selection $N_{2}^{1, \text { DDT }}<0$ yields a constant QCD background efficiency of $26 \%$ across the entire $\rho$ and $p_{T}$ range considered in this search. The chosen percentile maximizes the sensitivity to the Higgs boson signal. In order to select events in which the $H$ jet is most likely to contain two $b$ quarks, we use the double- $b$ tagger algorithm [61]. Several observables that characterize the distinct properties of $b$ hadrons and their flight directions in relation to the jet substructure are used as input variables to this multivariate algorithm in order to distinguish between $H$ jets and QCD jets. An $H$ jet is considered double- $b$ tagged if its double- $b$ tag discriminator value is above a threshold corresponding to a $1 \%$ misidentification rate for QCD jets and a 33\% efficiency for $H(b \bar{b})$ jets.

Events with (without) a double- $b$ tagged $H$ jet define the passing (failing) region. In the passing region, the gluon fusion process dominates, although other Higgs boson production mechanisms contribute: VBF (12\%), $V H$ (8\%), and $t \bar{t} H(5 \%)$. They are all taken into account when extracting the Higgs boson yield.

The contribution of $t \bar{t}$ production to the total SM background is estimated to be less than $3 \%$. It is obtained from simulation corrected with scale factors derived from a $t \bar{t}$-enriched control sample in which an isolated muon is required. This sample is included in a global fit used to extract the signal and the scale factors are treated as unconstrained parameters. They multiply the $t \bar{t}$ contribution, correcting its overall normalization and the double- $b$ mistag efficiency for jets originating from top quark decays.

The main background in the passing region, QCD multijet production, has a nontrivial jet mass shape that is difficult to model parametrically and dependent on jet $p_{T}$, so we constrain it using the signal-depleted failing region. Since the double- $b$ tagger discriminator and the jet mass are largely uncorrelated, the passing and failing regions have similar QCD jet mass distributions, and their ratio, the "pass-fail ratio" $R_{p / f}$, is expected to be nearly constant as a function of jet mass and $p_{T}$. To account for the residual difference between the shapes of passing and failing events, $R_{p / f}$ is parametrized as a polynomial in $\rho$ and $p_{T}, R_{p / f}\left(\rho, p_{T}\right)=\sum_{k, \ell} a_{k \ell} \rho^{k} p_{T}^{\ell}$. The coefficients $a_{k, \ell}$ have no external constraints but are determined from a simultaneous fit to the data in passing and failing regions across the whole jet mass range. To determine the order of the polynomial necessary to fit the data, a Fisher $F$-test [62] is performed. Based on its results, a polynomial of second order in $\rho$ and first order in $p_{T}$ is selected.

The systematic uncertainties associated with the jet mass scale, the jet mass resolution, and the $N_{2}^{1, \text { DDT }}$ selection efficiency are correlated among the $W, Z$, and $H(b \bar{b})$ processes. These uncertainties are estimated using an independent sample of merged $W$ jets. Additional details are available in the Supplemental Material [63], which includes Ref. [64]. The efficiency of the double- $b$ tagger is measured in data and simulation in a sample enriched in $b \bar{b}$ from gluon splitting [61]. Scale factors relating data and simulation are then computed and applied to the simulation. These scale factors determine the initial distributions of the jet mass for the $W(q \bar{q}), z(q \bar{q})$, and $H(b \bar{b})$ processes, and they are further constrained in the fit to data due to the presence of the $W$ and $Z$ resonances in the jet mass distribution. The uncertainty associated with the modeling of the GGF Higgs $p_{T}$ spectrum is propagated to the overall normalization of the GGF Higgs signal. In addition, the shape of the GGF Higgs $p_{T}$ distribution is allowed to vary depending on the Higgs boson $p_{T}$ by up to $30 \%$ at $1000 \mathrm{GeV}$, without changing the overall normalization. To account for some potentially $p_{T}$-dependent deviations due to missing higher-order corrections, uncertainties are applied to the $W(q \bar{q})$ and $Z(q \bar{q})$ yields that are $p_{T^{-}}$ dependent and correlated per $p_{T}$ bin. An additional 
TABLE I. Summary of the systematic uncertainties affecting the signal, $W$ and $Z+$ jets processes. Instances where the uncertainty does not apply are indicated by “...”.

\begin{tabular}{|c|c|c|}
\hline Systematic source & $W / Z$ & $H$ \\
\hline Integrated luminosity & $2.5 \%$ & $2.5 \%$ \\
\hline Trigger efficiency & $4 \%$ & $4 \%$ \\
\hline Pileup & $<1 \%$ & $<1 \%$ \\
\hline $\begin{array}{l}N_{2}^{1, \text { DDT }} \text { selection } \\
\text { efficiency }\end{array}$ & $4.3 \%$ & $4.3 \%$ \\
\hline Double- $b$ tag & $4 \%(Z)$ & $4 \%$ \\
\hline $\begin{array}{l}\text { Jet energy scale/ } \\
\text { resolution }\end{array}$ & $10 / 15 \%$ & $10 / 15 \%$ \\
\hline Jet mass scale $\left(p_{T}\right)$ & $0.4 \% / 100 \mathrm{GeV}\left(p_{T}\right)$ & $0.4 \% / 100 \mathrm{GeV}\left(p_{T}\right)$ \\
\hline $\begin{array}{l}\text { Simulation sample } \\
\text { size }\end{array}$ & $2-25 \%$ & 4-20\% (GGF) \\
\hline$H p_{T}$ correction & $\ldots$ & $30 \%(\mathrm{GGF})$ \\
\hline $\begin{array}{l}\text { NLO QCD } \\
\text { corrections }\end{array}$ & $10 \%$ & $\ldots$ \\
\hline $\begin{array}{l}\text { NLO EW } \\
\text { corrections }\end{array}$ & $15-35 \%$ & $\cdots$ \\
\hline $\begin{array}{r}\text { NLO EW } W / Z \\
\text { decorrelation }\end{array}$ & $5-15 \%$ & $\cdots$ \\
\hline
\end{tabular}

systematic uncertainty is included to account for potential differences between the $W$ and $Z$ higher-order corrections (EW $W / Z$ decorrelation). Finally, additional systematic uncertainties are applied to the $W(q \bar{q}), Z(q \bar{q})$, and $H(b \bar{b})$ yields to account for the uncertainties due to the jet energy scale and resolution [65], variations in the amount of pileup, and the integrated luminosity determination [66]. A quantitative summary of the systematic effects considered is shown in Table I.

In order to validate the background estimation method and associated systematic uncertainties, studies are performed on simulated samples injecting signal events and determining the bias on the measured signal cross section. No significant bias is observed in these studies.

A binned maximum likelihood fit to the observed $m_{\mathrm{SD}}$ distributions in the range 40 to $201 \mathrm{GeV}$ with $7 \mathrm{GeV}$ bin width is performed using the sum of the $H(b \bar{b}), W, Z, t \bar{t}$, and QCD multijet contributions. The fit is done simultaneously in the passing and failing regions of the six $p_{T}$ categories within $450<p_{T}<1000 \mathrm{GeV}$, and in the $t \bar{t}$ enriched control region. The production cross sections relative to the $\mathrm{SM}$ cross sections (signal strengths) for the Higgs and the $Z$ bosons, $\mu_{H}$ and $\mu_{Z}$, respectively, are extracted from the fit. Figure 1 shows the $m_{\mathrm{SD}}$ distributions in data for the passing and failing regions with measured SM background and $H(b \bar{b})$ contributions. Contributions from $W$ and $Z$ boson production are clearly visible in the data.

The measured $Z$ boson signal strength is $\mu_{Z}=$ $0.78 \pm 0.14(\text { stat })_{-0.13}^{+0.19}$ (syst), which corresponds to an
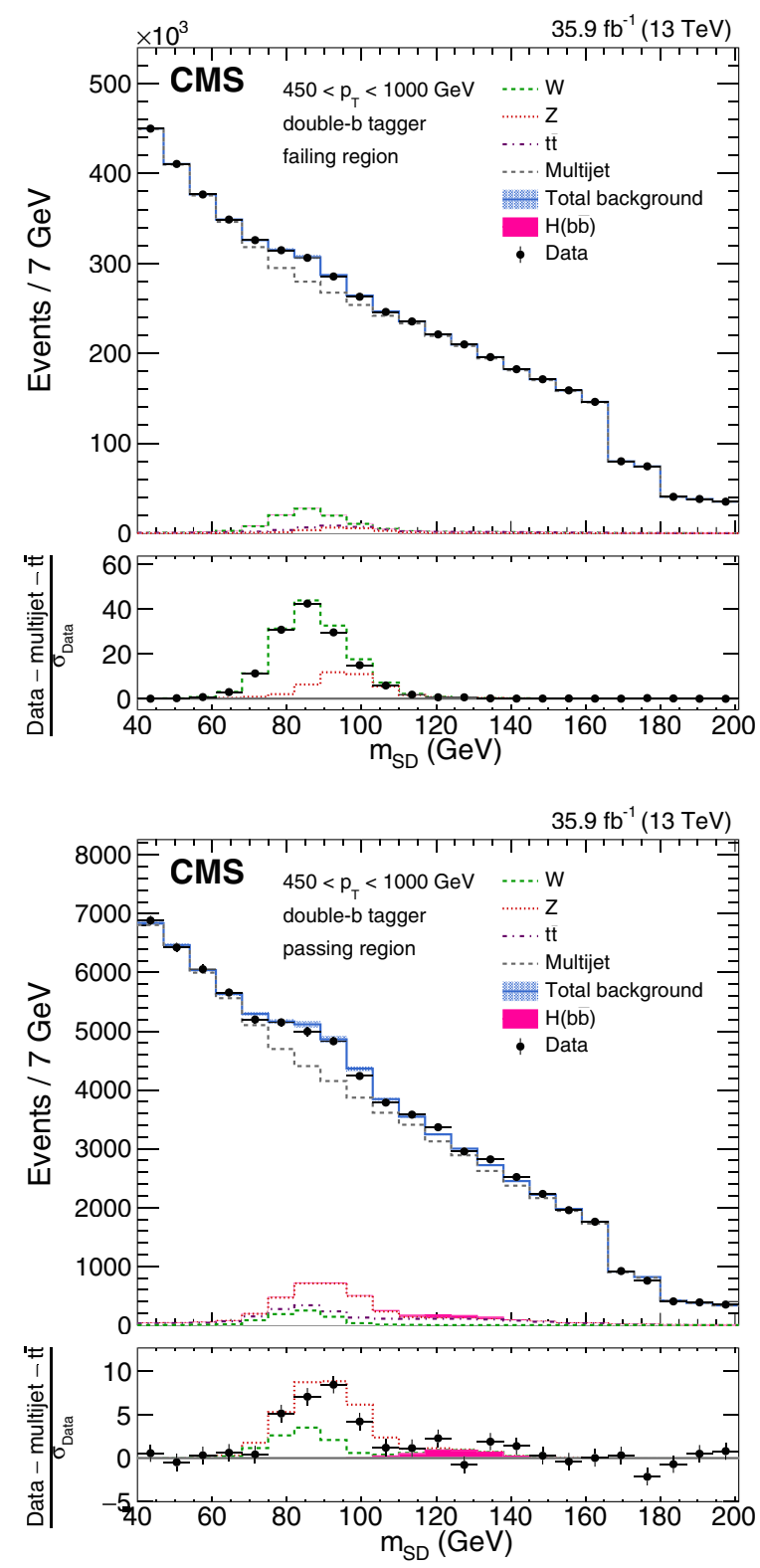

FIG. 1. The $m_{\mathrm{SD}}$ distributions in data for the failing (left) and passing (right) regions and combined $p_{T}$ categories. The QCD multijet background in the passing region is predicted using the failing region and the pass-fail ratio $R_{\mathrm{p} / \mathrm{f}}$. The features at 166 and $180 \mathrm{GeV}$ in the $m_{\mathrm{SD}}$ distribution are due to the kinematic selection on $\rho$, which affects each $p_{T}$ category differently. In the bottom panel, the ratio of the data to its statistical uncertainty, after subtracting the nonresonant backgrounds, is shown.

observed significance of 5.1 standard deviations $(\sigma)$ with $5.8 \sigma$ expected. This constitutes the first observation of the $Z$ boson signal in the single-jet topology [67] and validates the substructure and $b$ tagging techniques for the Higgs boson search in the same topology. The measured cross section for the $Z+$ jets process for jet $p_{T}>450 \mathrm{GeV}$ and $|\eta|<2.5$ is $0.85 \pm 0.16$ (stat) ${ }_{-0.14}^{+0.20}$ (syst) pb, which is 
TABLE II. Fitted signal strength, expected and observed significance of the Higgs and $Z$ boson signal. The $95 \%$ confidence level upper limit (UL) on the Higgs boson signal strength is also listed.

\begin{tabular}{lccc}
\hline \hline & \multicolumn{3}{c}{$H$ no $p_{T}$} \\
& $H$ & corrections & $Z$ \\
\hline Observed signal strength & $2.3_{-1.6}^{+1.8}$ & $3.2_{-2.0}^{+2.2}$ & $0.78_{-0.19}^{+0.23}$ \\
Expected UL signal strength & $<3.3$ & $<4.1$ & $\ldots$ \\
Observed UL signal strength & $<5.8$ & $<7.2$ & $\ldots$ \\
Expected significance & $0.7 \sigma$ & $0.5 \sigma$ & $5.8 \sigma$ \\
Observed significance & $1.5 \sigma$ & $1.6 \sigma$ & $5.1 \sigma$ \\
\hline \hline
\end{tabular}

consistent within uncertainties with the SM production cross section of $1.09 \pm 0.11 \mathrm{pb}$ [30]. Likewise, the measured Higgs boson signal strength is $\mu_{H}=2.3 \pm$ 1.5 (stat $)_{-0.4}^{+1.0}$ (syst) and includes the corrections to the Higgs boson $p_{T}$ spectrum described earlier. The corresponding observed (expected) upper limit on the Higgs boson signal strength at a 95\% confidence level is 5.8 (3.3), while the observed (expected) significance is $1.5 \sigma(0.7 \sigma)$. The observed $\mu_{H}$ implies a measured GGF cross section times $H(b \bar{b})$ branching fraction for jet $p_{T}>450 \mathrm{GeV}$ and $|\eta|<2.5$ of $74 \pm 48$ (stat) $)_{-10}^{+17}$ (syst) fb, assuming the SM values for the ratios of the different $H(b \bar{b})$ production modes. This measurement is consistent within uncertainties with the SM GGF cross section times $H(b \bar{b})$ branching fraction of $31.7 \pm 9.5 \mathrm{fb}$.

Table II summarizes the measured signal strengths and significances for the Higgs and $Z$ boson processes. In particular, they are also reported for the case in which no corrections to the Higgs boson $p_{T}$ spectrum are applied. Figure 2 shows the profile likelihood test statistic scan in data as function of the Higgs and $Z$ boson signal strengths $\left(\mu_{H}, \mu_{Z}\right)$.

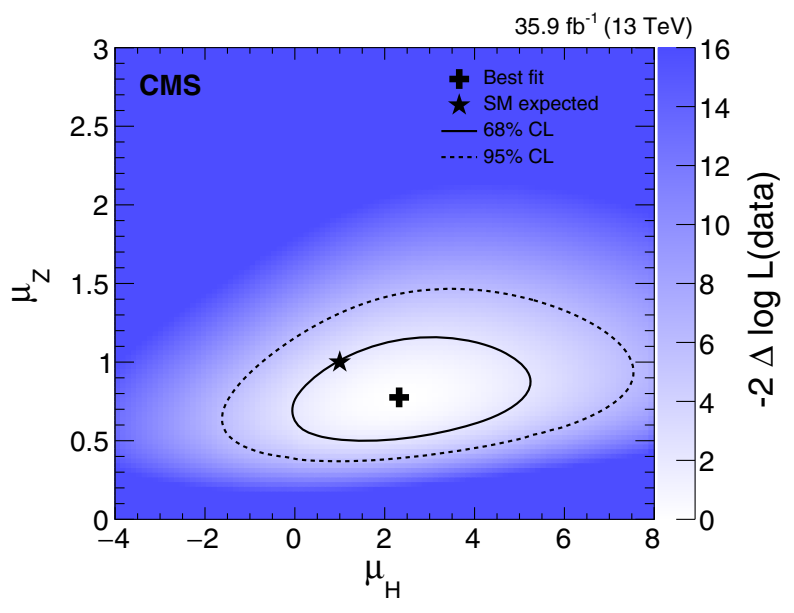

FIG. 2. Profile likelihood test statistic $-2 \Delta \log \mathcal{L}$ scan in data as a function of the Higgs and $Z$ bosons signal strengths $\left(\mu_{H}, \mu_{Z}\right)$.
In summary, an inclusive search for the standard model Higgs boson with $p_{T}>450 \mathrm{GeV}$ decaying to bottom quark-antiquark pairs and reconstructed as a single, large-radius jet is presented. The $Z+$ jets process is observed for the first time in the single-jet topology with a significance of 5.1 $\sigma$. The Higgs production is measured with an observed (expected) significance of $1.5 \sigma(0.7 \sigma)$ when including Higgs boson $p_{T}$ spectrum corrections accounting for higher-order and finite top quark mass effects. The measured cross section times branching fraction for the gluon fusion $H(b \bar{b})$ production for reconstructed $p_{T}$ and $|\eta|<2.5$ is $74 \pm 48(\text { stat })_{-10}^{+17}$ (syst) fb, which is consistent with the SM prediction within uncertainties.

We congratulate our colleagues in the CERN accelerator departments for the excellent performance of the LHC and thank the technical and administrative staffs at CERN and at other CMS institutes for their contributions to the success of the CMS effort. In addition, we gratefully acknowledge the computing centers and personnel of the Worldwide LHC Computing Grid for delivering so effectively the computing infrastructure essential to our analyses. Finally, we acknowledge the enduring support for the construction and operation of the LHC and the CMS detector provided by the following funding agencies: BMWFW and FWF (Austria); FNRS and FWO (Belgium); CNPq, CAPES, FAPERJ, and FAPESP (Brazil); MES (Bulgaria); CERN; CAS, MoST, and NSFC (China); COLCIENCIAS (Colombia); MSES and CSF (Croatia); RPF (Cyprus); SENESCYT (Ecuador); MoER, ERC IUT, and ERDF (Estonia); Academy of Finland, MEC, and HIP (Finland); CEA and CNRS/IN2P3 (France); BMBF, DFG, and HGF (Germany); GSRT (Greece); OTKA and NIH (Hungary); DAE and DST (India); IPM (Iran); SFI (Ireland); INFN (Italy); MSIP and NRF (Republic of Korea); LAS (Lithuania); MOE and UM (Malaysia); BUAP, CINVESTAV, CONACYT, LNS, SEP, and UASLP-FAI (Mexico); MBIE (New Zealand); PAEC (Pakistan); MSHE and NSC (Poland); FCT (Portugal); JINR (Dubna); MON, RosAtom, RAS, RFBR and RAEP (Russia); MESTD (Serbia); SEIDI, CPAN, PCTI and FEDER (Spain); Swiss Funding Agencies (Switzerland); MST (Taipei); ThEPCenter, IPST, STAR, and NSTDA (Thailand); TUBITAK and TAEK (Turkey); NASU and SFFR (Ukraine); STFC (United Kingdom); DOE and NSF (USA).

[1] A. Salam, Weak and electromagnetic interactions, in Elementary particle physics: relativistic groups and analyticity. Proceedings of the Eighth Nobel Symposium, edited by Nils Svartholm (Almqvist \& Wiksell, Stockholm, 1968), p. 367. 
[2] S. L. Glashow, Partial-Symmetries of Weak Interactions, Nucl. Phys. 22, 579 (1961).

[3] S. Weinberg, A Model of Leptons, Phys. Rev. Lett. 19, 1264 (1967).

[4] F. Englert and R. Brout, Broken Symmetry and the Mass of Gauge Vector Mesons, Phys. Rev. Lett. 13, 321 (1964).

[5] P. W. Higgs, Broken Symmetries, Massless particles and Gauge Fields, Phys. Rev. Lett. 12, 132 (1964).

[6] P. W. Higgs, Broken Symmetries and the Masses of Gauge Bosons, Phys. Rev. Lett. 13, 508 (1964).

[7] P. W. Higgs, Spontaneous Symmetry Breakdown without Massless Bosons, Phys. Rev. Lett. 145, 1156 (1966).

[8] G. S. Guralnik, C. R. Hagen, and T. W. B. Kibble, Global Conservation Laws and Massless Particles, Phys. Rev. Lett. 13, 585 (1964).

[9] ATLAS Collaboration, Observation of a new particle in the search for the Standard Model Higgs boson with the ATLAS detector at the LHC, Phys. Lett. B 716, 1 (2012).

[10] CMS Collaboration, Observation of a new boson at a mass of $125 \mathrm{GeV}$ with the CMS experiment at the LHC, Phys. Lett. B 716, 30 (2012).

[11] CMS Collaboration, Observation of a new boson with mass near $125 \mathrm{GeV}$ in pp collisions at $\sqrt{s}=7$ and $8 \mathrm{TeV}$, J. High Energy Phys. 06 (2013) 081.

[12] G. Aad et al. (ATLAS and CMS Collaborations), Measurements of the Higgs Boson Production and Decay Rates and Constraints on its Couplings from a Combined ATLAS and CMS Analysis of the LHC pp Collision Data at $\sqrt{s}=7$ and $8 \mathrm{TeV}$, J. High Energy Phys. 08 (2016) 045.

[13] D. de Florian et al., CERN Report No. CERN-2017-002-M, 2016.

[14] J. M. Butterworth, A. R. Davison, M. Rubin, and G. P. Salam, Jet Substructure as a New Higgs-Search Channel at the Large Hadron Collider, Phys. Rev. Lett. 100, 242001 (2008).

[15] C. Grojean, E. Salvioni, M. Schlaffer, and A. Weiler, Very boosted Higgs in gluon fusion, J. High Energy Phys. 05 (2014) 022.

[16] CMS Collaboration, The CMS experiment at the CERN LHC, J. Instrum. 3, S08004 (2008).

[17] S. Agostinelli et al. (GEANT4 Collaboration), GEANT4-A simulation toolkit, Nucl. Instrum. Methods Phys. Res., Sect. A 506, 250 (2003).

[18] J. Alwall, R. Frederix, S. Frixione, V. Hirschi, F. Maltoni, O. Mattelaer, H. S. Shao, T. Stelzer, P. Torrielli, and M. Zaro, The automated computation of tree-level and next-toleading order differential cross sections, and their matching to parton shower simulations, J. High Energy Phys. 07 (2014) 079.

[19] J. Alwall, S. S. Hoeche, F. Krauss, N. Lavesson, L. Lonnblad, F. Maltoni, M. L. Mangano, M. Moretti, C. G. Papadopoulos, F. Piccinini, S. Schumann, M. Treccani, J. Winter, and M. Worek, Comparative study of various algorithms for the merging of parton showers and matrix elements in hadronic collisions, Eur. Phys. J. C 53, 473 (2008).

[20] P. Nason, A new method for combining NLO QCD with shower Monte Carlo algorithms, J. High Energy Phys. 11 (2004) 040.
[21] S. Frixione, P. Nason, and C. Oleari, Matching NLO QCD computations with parton shower simulations: the POWHEG method, J. High Energy Phys. 11 (2007) 070.

[22] S. Alioli, P. Nason, C. Oleari, and E. Re, A general framework for implementing NLO calculations in shower Monte Carlo programs: the POWHEG BOX, J. High Energy Phys. 06 (2010) 043.

[23] T. Sjöstrand, S. Ask, J. R. Christiansen, R. Corke, N. Desai, P. Ilten, S. Mrenna, S. Prestel, C. O. Rasmussen, and P.Z. Skands, An introduction to PYthiA 8.2, Comput. Phys. Commun. 191, 159 (2015).

[24] CMS Collaboration, Event generator tunes obtained from underlying event and multiparton scattering measurements, Eur. Phys. J. C 76, 155 (2016).

[25] J. M. Campbell and R. K. Ellis, MCFM for the Tevatron and the LHC, Nucl. Phys. B, Proc. Suppl. 205-206, 10 (2010).

[26] M. Czakon, P. Fiedler, and A. Mitov, Total Top-Quark PairProduction Cross Section at Hadron Colliders Through $\mathrm{O}\left(\alpha_{S}^{4}\right)$, Phys. Rev. Lett. 110, 252004 (2013).

[27] S. Kallweit, J. M. Lindert, P. Maierhöfer, S. Pozzorini, and M. Schönherr, NLO electroweak automation and precise predictions for $\mathrm{W}+$ multijet production at the LHC, J. High Energy Phys. 04 (2015) 012.

[28] S. Kallweit, J. M. Lindert, P. Maierhöfer, S. Pozzorini, and M. Schönherr, NLO QCD + EW predictions for $\mathrm{V}+$ jets including off-shell vector-boson decays and multijet merging, J. High Energy Phys. 04 (2016) 021.

[29] S. Kallweit, J. M. Lindert, S. Pozzorini, M. Schönherr, and P. Maierhöfer, NLO QCD + EW automation and precise predictions for $\mathrm{V}+$ multijet production, arXiv:1505.05704.

[30] J. M. Lindert et al., Precise predictions for $\mathrm{V}+$ jets dark matter backgrounds, Eur. Phys. J. C 77, 829 (2017).

[31] R. D. Ball et al. (NNPDF Collaboration), Parton distributions for the LHC run II, J. High Energy Phys. 04 (2015) 040 .

[32] D. de Florian, G. Ferrera, M. Grazzini, and D. Tommasini, Higgs boson production at the LHC: Transverse momentum resummation effects in the $\mathrm{H} \rightarrow 2 \gamma, \mathrm{H} \rightarrow \mathrm{WW} \rightarrow \ell \nu \ell \nu$ and $\mathrm{H} \rightarrow \mathrm{ZZ} \rightarrow 41$ decay modes, J. High Energy Phys. 06 (2012) 132.

[33] M. Grazzini and H. Sargsyan, Heavy-quark mass effects in Higgs boson production at the LHC, J. High Energy Phys. 09 (2013) 129.

[34] E. Bagnaschi, G. Degrassi, P. Slavich, and A. Vicini, Higgs production via gluon fusion in the POWHEG approach in the SM and in the MSSM, J. High Energy Phys. 02 (2012) 088.

[35] E. Bagnaschi and A. Vicini, The Higgs transverse momentum distribution in gluon fusion as a multiscale problem, J. High Energy Phys. 01 (2016) 056.

[36] U. J. Baur and E. W. N. Glover, Higgs boson production at large transverse momentum in hadronic collisions, Nucl. Phys. B339, 38 (1990).

[37] R. Boughezal, F. Caola, K. Melnikov, F. Petriello, and M. Schulze, Higgs boson production in association with a jet at next-to-next-to-leading order in perturbative QCD, J. High Energy Phys. 06 (2013) 072.

[38] R. Boughezal, C. Focke, W. Giele, X. Liu, and F. Petriello, Higgs boson production in association with a jet using jettiness subtraction, Phys. Lett. B 748, 5 (2015). 
[39] R. Boughezal, J. M. Campbell, R. K. Ellis, C. Focke, W. Giele, X. Liu, F. Petriello, and C. Williams, Color-singlet production at NNLO in MCFM, Eur. Phys. J. C 77, 7 (2017).

[40] X. Chen, T. Gehrmann, N. Glover, and M. Jaquier, Higgs plus one jet production at NNLO, Proc. Sci., RADCOR2015 (2016) 056.

[41] S. Catani, F. Krauss, R. Kuhn, and B. R. Webber, QCD matrix Elements + Parton showers, J. High Energy Phys. 11 (2001) 063.

[42] L. Lonnblad, Correcting the colour-dipole cascade model with fixed order matrix elements, J. High Energy Phys. 05 (2002) 046.

[43] V. Hirschi and O. Mattelaer, Automated event generation for loop-induced processes, J. High Energy Phys. 10 (2015) 146.

[44] M. Buschmann, D. Goncalves, S. Kuttimalai, M. Schonherr, F. Krauss, and T. Plehn, Mass effects in the Higgs-gluon coupling: Boosted vs. off-shell production, J. High Energy Phys. 02 (2015) 038.

[45] N. Greiner, S. Höche, G. Luisoni, M. Schönherr, J. Winter, and V. Yundin, Phenomenological analysis of Higgs boson production through gluon fusion in association with jets, J. High Energy Phys. 01 (2016) 169.

[46] T. Neumann and C. Williams, Higgs bosons at high $p_{\mathrm{T}}$, Phys. Rev. D 95, 014004 (2017).

[47] N. Greiner, S. Höche, G. Luisoni, M. Schönherr, and J. Winter, Full mass dependence in Higgs boson production in association with jets at the LHC and FCC, J. High Energy Phys. 01 (2017) 091.

[48] M. Cacciari, F. A. Dreyer, A. Karlberg, G. P. Salam, and G. Zanderighi, Fully Differential Vector-Boson-Fusion Higgs Production at Next-to-Next-to-Leading Order, Phys. Rev. Lett. 115, 082002 (2015).

[49] F. A. Dreyer and A. Karlberg, Vector-Boson Fusion Higgs Production at Three Loops in QCD, Phys. Rev. Lett. 117, 072001 (2016).

[50] CMS Collaboration, Particle-flow reconstruction and global event description with the CMS detector, J. Instrum. 12, P10003 (2017).

[51] M. Cacciari, G. P. Salam, and G. Soyez, The anti- $k_{t}$ jet clustering algorithm, J. High Energy Phys. 04 (2008) 063.

[52] D. Bertolini, P. Harris, M. Low, and N. Tran, Pileup per particle identification, J. High Energy Phys. 10 (2014) 059.
[53] D. Krohn, J. Thaler, and L. Wang, Jet trimming, J. High Energy Phys. 02 (2010) 084.

[54] M. Dasgupta, A. Fregoso, S. Marzani, and G. P. Salam, Towards an understanding of jet substructure, J. High Energy Phys. 09 (2013) 029.

[55] A. J. Larkoski, S. Marzani, G. Soyez, and J. Thaler, Soft drop, J. High Energy Phys. 05 (2014) 146.

[56] CMS Collaboration, CMS Physics Analysis Summary Report No. CMS-PAS-JME-16-003, 2017.

[57] J. Dolen, P. Harris, S. Marzani, S. Rappoccio, and N. Tran, Thinking outside the ROCs: Designing Decorrelated Taggers (DDT) for jet substructure, J. High Energy Phys. 05 (2016) 156.

[58] I. Moult, L. Necib, and J. Thaler, New angles on energy correlation functions, J. High Energy Phys. 12 (2016) 153.

[59] A. J. Larkoski, G. P. Salam, and J. Thaler, Energy correlation functions for jet substructure, J. High Energy Phys. 06 (2013) 108.

[60] J. Thaler and K. Van Tilburg, Identifying boosted objects with N-subjettiness, J. High Energy Phys. 03 (2011) 015.

[61] CMS Collaboration, Identification of Double-b Quark Jets in Boosted Event Topologies, CMS Physics Analysis Summary CMS-PAS-BTV-15-002 (CERN, Geneva, 2016).

[62] R. A. Fisher, On the interpretation of $\chi^{2}$ from contingency tables, and the calculation of P, J. R. Stat. Soc. 85, 87 (1922).

[63] See Supplemental Material at http://link.aps.org/ supplemental/10.1103/PhysRevLett.120.071802 for details on the systematic uncertainties associated with the jet mass scale, the jet mass resolution, and the $N_{2}^{1, \text { DDT }}$ selection.

[64] M. Bahr, S. Gieseke, M. A. Gigg, D. Grellscheid, K. Hamilton, O. Latunde-Dada, S. Platzer, P. Richardson, M. H. Seymour, A. Sherstnev, J. Tully, and B. R. Webber, Herwig ++ physics and manual, Eur. Phys. J. C 58, 639 (2008).

[65] CMS Collaboration, Determination of jet energy calibration and transverse momentum resolution in CMS, J. Instrum. 6, P11002 (2011).

[66] CMS Collaboration, CMS Physics Analysis Summary Report No. CMS-PAS-LUM-17-001 2017.

[67] ATLAS Collaboration, Measurement of the cross-section of high transverse momentum vector bosons reconstructed as single jets and studies of jet substructure in $p p$ collisions at $\sqrt{s}=7 \mathrm{TeV}$ with the ATLAS detector, New J. Phys. 16, 113013 (2014).

A. M. Sirunyan, ${ }^{1}$ A. Tumasyan, ${ }^{1}$ W. Adam, ${ }^{2}$ F. Ambrogi, ${ }^{2}$ E. Asilar, ${ }^{2}$ T. Bergauer, ${ }^{2}$ J. Brandstetter, ${ }^{2}$ E. Brondolin, ${ }^{2}$ M. Dragicevic, ${ }^{2}$ J. Erö, ${ }^{2}$ M. Flechl, ${ }^{2}$ M. Friedl, ${ }^{2}$ R. Frühwirth, ${ }^{2, b}$ V. M. Ghete, ${ }^{2}$ J. Grossmann, ${ }^{2}$ J. Hrubec, ${ }^{2}$ M. Jeitler, ${ }^{2, b}$ A. König, ${ }^{2}$ N. Krammer, ${ }^{2}$ I. Krätschmer, ${ }^{2}$ D. Liko, ${ }^{2}$ T. Madlener, ${ }^{2}$ I. Mikulec, ${ }^{2}$ E. Pree, ${ }^{2}$ N. Rad, ${ }^{2}$ H. Rohringer, ${ }^{2}$ J. Schieck, ${ }^{2, b}$ R. Schöfbeck, ${ }^{2}$ M. Spanring, ${ }^{2}$ D. Spitzbart, ${ }^{2}$ W. Waltenberger, ${ }^{2}$ J. Wittmann, ${ }^{2}$ C.-E. Wulz, ${ }^{2, b}$ M. Zarucki, ${ }^{2}$ V. Chekhovsky, ${ }^{3}$ Y. Dydyshka, ${ }^{3}$ J. Suarez Gonzalez, ${ }^{3}$ E. A. De Wolf, ${ }^{4}$ D. Di Croce, ${ }^{4}$ X. Janssen, ${ }^{4}$ J. Lauwers, ${ }^{4}$ M. Van De Klundert, ${ }^{4}$ H. Van Haevermaet, ${ }^{4}$ P. Van Mechelen, ${ }^{4}$ N. Van Remortel, ${ }^{4}$ S. Abu Zeid, ${ }^{5}$ F. Blekman, ${ }^{5}$ J. D'Hondt, ${ }^{5}$ I. De Bruyn, ${ }^{5}$ J. De Clercq, ${ }^{5}$ K. Deroover, ${ }^{5}$ G. Flouris, ${ }^{5}$ D. Lontkovskyi, ${ }^{5}$ S. Lowette, ${ }^{5}$ S. Moortgat, ${ }^{5}$ L. Moreels, ${ }^{5}$ Q. Python, ${ }^{5}$ K. Skovpen, ${ }^{5}$ S. Tavernier, ${ }^{5}$ W. Van Doninck, ${ }^{5}$ P. Van Mulders, ${ }^{5}$ I. Van Parijs, ${ }^{5}$ D. Beghin, ${ }^{6}$ H. Brun, ${ }^{6}$ B. Clerbaux, ${ }^{6}$ G. De Lentdecker, ${ }^{6}$ H. Delannoy, ${ }^{6}$ B. Dorney, ${ }^{6}$ G. Fasanella, ${ }^{6}$ L. Favart, ${ }^{6}$ R. Goldouzian, ${ }^{6}$ A. Grebenyuk, ${ }^{6}$ G. Karapostoli, ${ }^{6}$ T. Lenzi, ${ }^{6}$ J. Luetic, ${ }^{6}$ T. Maerschalk, ${ }^{6}$ A. Marinov, ${ }^{6}$ A. Randle-conde, ${ }^{6}$ T. Seva, ${ }^{6}$ E. Starling, ${ }^{6}$ 
C. Vander Velde, ${ }^{6}$ P. Vanlaer, ${ }^{6}$ D. Vannerom, ${ }^{6}$ R. Yonamine, ${ }^{6}$ F. Zenoni, ${ }^{6}$ F. Zhang, ${ }^{6, c}$ A. Cimmino, ${ }^{7}$ T. Cornelis, ${ }^{7}$ D. Dobur, ${ }^{7}$ A. Fagot, ${ }^{7}$ M. Gul, ${ }^{7}$ I. Khvastunov, ${ }^{7, d}$ D. Poyraz, ${ }^{7}$ C. Roskas, ${ }^{7}$ S. Salva, ${ }^{7}$ M. Tytgat, ${ }^{7}$ W. Verbeke, ${ }^{7}$ N. Zaganidis, ${ }^{7}$ H. Bakhshiansohi, ${ }^{8}$ O. Bondu, ${ }^{8}$ S. Brochet, ${ }^{8}$ G. Bruno, ${ }^{8}$ C. Caputo, ${ }^{8}$ A. Caudron, ${ }^{8}$ P. David, ${ }^{8}$ S. De Visscher, ${ }^{8}$ C. Delaere, ${ }^{8}$ M. Delcourt, ${ }^{8}$ B. Francois, ${ }^{8}$ A. Giammanco, ${ }^{8}$ M. Komm, ${ }^{8}$ G. Krintiras, ${ }^{8}$ V. Lemaitre, ${ }^{8}$ A. Magitteri, ${ }^{8}$ A. Mertens, ${ }^{8}$ M. Musich, ${ }^{8}$ K. Piotrzkowski, ${ }^{8}$ L. Quertenmont, ${ }^{8}$ A. Saggio, ${ }^{8}$ M. Vidal Marono, ${ }^{8}$ S. Wertz, ${ }^{8}$ J. Zobec, ${ }^{8}$ N. Beliy, ${ }^{9}$ W. L. Aldá Júnior, ${ }^{10}$ F. L. Alves,${ }^{10}$ G. A. Alves,${ }^{10}$ L. Brito, ${ }^{10}$ M. Correa Martins Junior, ${ }^{10}$ C. Hensel,${ }^{10}$ A. Moraes, ${ }^{10}$ M. E. Pol,${ }^{10}$ P. Rebello Teles,${ }^{10}$ E. Belchior Batista Das Chagas, ${ }^{11}$ W. Carvalho, ${ }^{11}$ J. Chinellato,${ }^{11, e}$ E. Coelho, ${ }^{11}$ E. M. Da Costa, ${ }^{11}$ G. G. Da Silveira, ${ }^{11, f}$ D. De Jesus Damiao, ${ }^{11}$ S. Fonseca De Souza, ${ }^{11}$ L. M. Huertas Guativa, ${ }^{11}$ H. Malbouisson, ${ }^{11}$ M. Melo De Almeida, ${ }^{11}$ C. Mora Herrera, ${ }^{11}$ L. Mundim, ${ }^{11}$ H. Nogima, ${ }^{11}$ L. J. Sanchez Rosas, ${ }^{11}$ A. Santoro, ${ }^{11}$ A. Sznajder, ${ }^{11}$ M. Thiel, ${ }^{11}$ E. J. Tonelli Manganote, ${ }^{11, \mathrm{e}}$ F. Torres Da Silva De Araujo, ${ }^{11}$ A. Vilela Pereira, ${ }^{11}$ S. Ahuja, ${ }^{12 \mathrm{a}}$ C. A. Bernardes, ${ }^{12 \mathrm{a}}$ T. R. Fernandez Perez Tomei, ${ }^{12 \mathrm{a}}$ E. M. Gregores, ${ }^{12 \mathrm{~b}}$ P. G. Mercadante, ${ }^{12 \mathrm{~b}}$ S. F. Novaes, ${ }^{12 \mathrm{a}}$

Sandra S. Padula, ${ }^{12 \mathrm{a}}$ D. Romero Abad, ${ }^{12 \mathrm{~b}}$ J. C. Ruiz Vargas, ${ }^{12 \mathrm{a}}$ A. Aleksandrov,${ }^{13}$ R. Hadjiiska, ${ }^{13}$ P. Iaydjiev, ${ }^{13}$ M. Misheva, ${ }^{13}$ M. Rodozov, ${ }^{13}$ M. Shopova, ${ }^{13}$ G. Sultanov, ${ }^{13}$ A. Dimitrov ${ }^{14}$ I. Glushkov, ${ }^{14}$ L. Litov, ${ }^{14}$ B. Pavlov, ${ }^{14}$ P. Petkov, ${ }^{14}$ W. Fang, ${ }^{15,9}$ X. Gao, ${ }^{15, g}$ L. Yuan, ${ }^{15}$ M. Ahmad, ${ }^{16}$ J. G. Bian, ${ }^{16}$ G. M. Chen, ${ }^{16}$ H. S. Chen, ${ }^{16}$ M. Chen, ${ }^{16}$ Y. Chen, ${ }^{16}$ C. H. Jiang, ${ }^{16}$ D. Leggat, ${ }^{16}$ H. Liao, ${ }^{16}$ Z. Liu, ${ }^{16}$ F. Romeo, ${ }^{16}$ S. M. Shaheen, ${ }^{16}$ A. Spiezia, ${ }^{16}$ J. Tao, ${ }^{16}$ C. Wang, ${ }^{16}$ Z. Wang, ${ }^{16}$ E. Yazgan, ${ }^{16}$ H. Zhang, ${ }^{16}$ S. Zhang, ${ }^{16}$ J. Zhao, ${ }^{16}$ Y. Ban,${ }^{17}$ G. Chen, ${ }^{17}$ Q. Li, ${ }^{17}$ S. Liu,${ }^{17}$ Y. Mao, ${ }^{17}$ S. J. Qian, ${ }^{17}$

D. Wang, ${ }^{17}$ Z. Xu, ${ }^{17}$ C. Avila, ${ }^{18}$ A. Cabrera, ${ }^{18}$ L. F. Chaparro Sierra,${ }^{18}$ C. Florez,${ }^{18}$ C. F. González Hernández, ${ }^{18}$ J. D. Ruiz Alvarez, ${ }^{18}$ B. Courbon, ${ }^{19}$ N. Godinovic, ${ }^{19}$ D. Lelas, ${ }^{19}$ I. Puljak, ${ }^{19}$ P. M. Ribeiro Cipriano, ${ }^{19}$ T. Sculac, ${ }^{19}$ Z. Antunovic, ${ }^{20}$ M. Kovac, ${ }^{20}$ V. Brigljevic, ${ }^{21}$ D. Ferencek, ${ }^{21}$ K. Kadija, ${ }^{21}$ B. Mesic, ${ }^{21}$ A. Starodumov, ${ }^{21, \mathrm{~h}}$ T. Susa, ${ }^{21}$ M. W. Ather, ${ }^{22}$ A. Attikis, ${ }^{22}$ G. Mavromanolakis, ${ }^{22}$ J. Mousa, ${ }^{22}$ C. Nicolaou, ${ }^{22}$ F. Ptochos, ${ }^{22}$ P. A. Razis, ${ }^{22}$ H. Rykaczewski, ${ }^{22}$ M. Finger, ${ }^{23, \mathrm{i}}$ M. Finger Jr., ${ }^{23, \mathrm{i}}$ E. Carrera Jarrin, ${ }^{24}$ Y. Assran, ${ }^{25, \mathrm{j}, \mathrm{k}}$ M. A. Mahmoud,${ }^{25,1, \mathrm{k}}$ A. Mahrous, ${ }^{25, \mathrm{~m}}$ R. K. Dewanjee, ${ }^{26}$ M. Kadastik, ${ }^{26}$ L. Perrini, ${ }^{26}$ M. Raidal, ${ }^{26}$ A. Tiko, ${ }^{26}$ C. Veelken, ${ }^{26}$ P. Eerola, ${ }^{27}$ H. Kirschenmann, ${ }^{27}$ J. Pekkanen, ${ }^{27}$ M. Voutilainen, ${ }^{27}$ T. Järvinen, ${ }^{28}$ V. Karimäki, ${ }^{28}$ R. Kinnunen, ${ }^{28}$ T. Lampén, ${ }^{28}$ K. Lassila-Perini, ${ }^{28}$ S. Lehti, ${ }^{28}$ T. Lindén, ${ }^{28}$ P. Luukka, ${ }^{28}$ E. Tuominen, ${ }^{28}$ J. Tuominiemi, ${ }^{28}$ J. Talvitie, ${ }^{29}$ T. Tuuva, ${ }^{29}$ M. Besancon, ${ }^{30}$ F. Couderc, ${ }^{30}$ M. Dejardin, ${ }^{30}$ D. Denegri, ${ }^{30}$ J. L. Faure, ${ }^{30}$ F. Ferri, ${ }^{30}$ S. Ganjour, ${ }^{30}$ S. Ghosh ${ }^{30}$ A. Givernaud, ${ }^{30}$ P. Gras,${ }^{30}$ G. Hamel de Monchenault,${ }^{30}$ P. Jarry,${ }^{30}$ I. Kucher, ${ }^{30}$ C. Leloup, ${ }^{30}$ E. Locci, ${ }^{30}$ M. Machet,${ }^{30}$ J. Malcles, ${ }^{30}$ G. Negro, ${ }^{30}$ J. Rander, ${ }^{30}$ A. Rosowsky, ${ }^{30}$ M. Ö. Sahin, ${ }^{30}$ M. Titov, ${ }^{30}$ A. Abdulsalam, ${ }^{31}$ C. Amendola, ${ }^{31}$ I. Antropov, ${ }^{31}$ S. Baffioni, ${ }^{31}$ F. Beaudette, ${ }^{31}$ P. Busson, ${ }^{31}$ L. Cadamuro, ${ }^{31}$ C. Charlot, ${ }^{31}$ R. Granier de Cassagnac, ${ }^{31}$ M. Jo, ${ }^{31}$ S. Lisniak, ${ }^{31}$ A. Lobanov,${ }^{31}$ J. Martin Blanco, ${ }^{31}$ M. Nguyen, ${ }^{31}$ C. Ochando, ${ }^{31}$ G. Ortona, ${ }^{31}$ P. Paganini, ${ }^{31}$ P. Pigard, ${ }^{31}$ R. Salerno, ${ }^{31}$ J. B. Sauvan, ${ }^{31}$ Y. Sirois, ${ }^{31}$ A. G. Stahl Leiton, ${ }^{31}$ T. Strebler, ${ }^{31}$ Y. Yilmaz, ${ }^{31}$ A. Zabi, ${ }^{31}$ A. Zghiche, ${ }^{31}$ J.-L. Agram, ${ }^{32, \mathrm{n}}$ J. Andrea,${ }^{32}$ D. Bloch,${ }^{32}$ J.-M. Brom, ${ }^{32}$ M. Buttignol, ${ }^{32}$ E. C. Chabert, ${ }^{32}$ N. Chanon, ${ }^{32}$ C. Collard ${ }^{32}$ E. Conte, ${ }^{32, n}$ X. Coubez, ${ }^{32}$ J.-C. Fontaine, ${ }^{32, n}$ D. Gelé, ${ }^{32}$ U. Goerlach, ${ }^{32}$ M. Jansová, ${ }^{32}$ A.-C. Le Bihan, ${ }^{32}$ N. Tonon, ${ }^{32}$ P. Van Hove, ${ }^{32}$ S. Gadrat,${ }^{33}$ S. Beauceron, ${ }^{34}$ C. Bernet, ${ }^{34}$ G. Boudoul,${ }^{34}$ R. Chierici,${ }^{34}$ D. Contardo,${ }^{34}$ P. Depasse, ${ }^{34}$ H. El Mamouni, ${ }^{34}$ J. Fay,${ }^{34}$ L. Finco, ${ }^{34}$ S. Gascon, ${ }^{34}$ M. Gouzevitch, ${ }^{34}$ G. Grenier, ${ }^{34}$ B. Ille, ${ }^{34}$ F. Lagarde, ${ }^{34}$ I. B. Laktineh, ${ }^{34}$ M. Lethuillier, ${ }^{34}$ L. Mirabito, ${ }^{34}$ A. L. Pequegnot, ${ }^{34}$ S. Perries,${ }^{34}$ A. Popov, ${ }^{34,0}$ V. Sordini,${ }^{34}$ M. Vander Donckt, ${ }^{34}$ S. Viret, ${ }^{34}$ A. Khvedelidze, ${ }^{35, i}$ Z. Tsamalaidze, ${ }^{36, i}$ C. Autermann, ${ }^{37}$ L. Feld, ${ }^{37}$ M. K. Kiesel, ${ }^{37}$ K. Klein, ${ }^{37}$ M. Lipinski, ${ }^{37}$ M. Preuten, ${ }^{37}$ C. Schomakers, ${ }^{37}$ J. Schulz, ${ }^{37}$ T. Verlage, ${ }^{37}$ V. Zhukov, ${ }^{37,0}$ A. Albert, ${ }^{38}$ E. Dietz-Laursonn, ${ }^{38}$ D. Duchardt, ${ }^{38}$ M. Endres, ${ }^{38}$ M. Erdmann, ${ }^{38}$ S. Erdweg, ${ }^{38}$

T. Esch, ${ }^{38}$ R. Fischer, ${ }^{38}$ A. Güth, ${ }^{38}$ M. Hamer, ${ }^{38}$ T. Hebbeker, ${ }^{38}$ C. Heidemann, ${ }^{38}$ K. Hoepfner, ${ }^{38}$ S. Knutzen, ${ }^{38}$

M. Merschmeyer, ${ }^{38}$ A. Meyer, ${ }^{38}$ P. Millet, ${ }^{38}$ S. Mukherjee, ${ }^{38}$ T. Pook, ${ }^{38}$ M. Radziej, ${ }^{38}$ H. Reithler, ${ }^{38}$ M. Rieger, ${ }^{38}$ F. Scheuch, ${ }^{38}$ D. Teyssier ${ }^{38}$ S. Thüer, ${ }^{38}$ G. Flügge, ${ }^{39}$ B. Kargoll,${ }^{39}$ T. Kress, ${ }^{39}$ A. Künsken, ${ }^{39}$ J. Lingemann, ${ }^{39}$ T. Müller, ${ }^{39}$ A. Nehrkorn, ${ }^{39}$ A. Nowack, ${ }^{39}$ C. Pistone, ${ }^{39}$ O. Pooth, ${ }^{39}$ A. Stahl, ${ }^{39, p}$ M. Aldaya Martin, ${ }^{40}$ T. Arndt, ${ }^{40}$

C. Asawatangtrakuldee, ${ }^{40}$ K. Beernaert, ${ }^{40}$ O. Behnke, ${ }^{40}$ U. Behrens, ${ }^{40}$ A. Bermúdez Martínez, ${ }^{40}$ A. A. Bin Anuar, ${ }^{40}$ K. Borras, ${ }^{40, q}$ V. Botta, ${ }^{40}$ A. Campbell, ${ }^{40}$ P. Connor, ${ }^{40}$ C. Contreras-Campana,${ }^{40}$ F. Costanza,${ }^{40}$ C. Diez Pardos,${ }^{40}$ G. Eckerlin, ${ }^{40}$ D. Eckstein, ${ }^{40}$ T. Eichhorn, ${ }^{40}$ E. Eren, ${ }^{40}$ E. Gallo, ${ }^{40, r}$ J. Garay Garcia ${ }^{40}$ A. Geiser, ${ }^{40}$ A. Gizhko, ${ }^{40}$ J. M. Grados Luyando, ${ }^{40}$ A. Grohsjean, ${ }^{40}$ P. Gunnellini, ${ }^{40}$ M. Guthoff, ${ }^{40}$ A. Harb, ${ }^{40}$ J. Hauk, ${ }^{40}$ M. Hempel, ${ }^{40, s}$ H. Jung ${ }^{40}$ A. Kalogeropoulos, ${ }^{40}$ M. Kasemann, ${ }^{40}$ J. Keaveney, ${ }^{40}$ C. Kleinwort, ${ }^{40}$ I. Korol, ${ }^{40}$ D. Krücker, ${ }^{40}$ W. Lange, ${ }^{40}$ A. Lelek, ${ }^{40}$ T. Lenz, ${ }^{40}$ J. Leonard ${ }^{40}$ K. Lipka, ${ }^{40}$ W. Lohmann, ${ }^{40, s}$ R. Mankel, ${ }^{40}$ I.-A. Melzer-Pellmann, ${ }^{40}$ A. B. Meyer, ${ }^{40}$ G. Mittag, ${ }^{40}$ J. Mnich,${ }^{40}$ A. Mussgiller, ${ }^{40}$ E. Ntomari, ${ }^{40}$ D. Pitzl, ${ }^{40}$ A. Raspereza, ${ }^{40}$ B. Roland, ${ }^{40}$ M. Savitskyi,${ }^{40}$ P. Saxena, ${ }^{40}$ R. Shevchenko, ${ }^{40}$ S. Spannagel, ${ }^{40}$ N. Stefaniuk, ${ }^{40}$ G. P. Van Onsem, ${ }^{40}$ R. Walsh, ${ }^{40}$ Y. Wen, ${ }^{40}$ K. Wichmann, ${ }^{40}$ C. Wissing, ${ }^{40}$ 
O. Zenaiev, ${ }^{40}$ R. Aggleton, ${ }^{41}$ S. Bein, ${ }^{41}$ V. Blobel, ${ }^{41}$ M. Centis Vignali, ${ }^{41}$ T. Dreyer, ${ }^{41}$ E. Garutti, ${ }^{41}$ D. Gonzalez, ${ }^{41}$ J. Haller, ${ }^{41}$ A. Hinzmann, ${ }^{41}$ M. Hoffmann, ${ }^{41}$ A. Karavdina, ${ }^{41}$ R. Klanner, ${ }^{41}$ R. Kogler,${ }^{41}$ N. Kovalchuk,${ }^{41}$ S. Kurz, ${ }^{41}$ T. Lapsien, ${ }^{41}$ I. Marchesini ${ }^{41}$ D. Marconi, ${ }^{41}$ M. Meyer, ${ }^{41}$ M. Niedziela, ${ }^{41}$ D. Nowatschin, ${ }^{41}$ F. Pantaleo, ${ }^{41, p}$ T. Peiffer, ${ }^{41}$ A. Perieanu, ${ }^{41}$ C. Scharf, ${ }^{41}$ P. Schleper, ${ }^{41}$ A. Schmidt, ${ }^{41}$ S. Schumann, ${ }^{41}$ J. Schwandt, ${ }^{41}$ J. Sonneveld, ${ }^{41}$ H. Stadie, ${ }^{41}$ G. Steinbrück, ${ }^{41}$ F. M. Stober, ${ }^{41}$ M. Stöver, ${ }^{41}$ H. Tholen, ${ }^{41}$ D. Troendle, ${ }^{41}$ E. Usai ${ }^{41}$ L. Vanelderen, ${ }^{41}$ A. Vanhoefer, ${ }^{41}$ B. Vormwald ${ }^{41}$ M. Akbiyik,${ }^{42}$ C. Barth ${ }^{42}$ S. Baur, ${ }^{42}$ E. Butz,${ }^{42}$ R. Caspart ${ }^{42}$ T. Chwalek,${ }^{42}$ F. Colombo, ${ }^{42}$ W. De Boer,${ }^{42}$ A. Dierlamm, ${ }^{42}$ B. Freund, ${ }^{42}$ R. Friese, ${ }^{42}$ M. Giffels, ${ }^{42}$ D. Haitz ${ }^{42}$ M. A. Harrendorf, ${ }^{42}$ F. Hartmann, ${ }^{42, p}$ S. M. Heindl, ${ }^{42}$ U. Husemann, ${ }^{42}$ F. Kassel, ${ }^{42, p}$ S. Kudella, ${ }^{42}$ H. Mildner, ${ }^{42}$ M. U. Mozer, ${ }^{42}$ Th. Müller, ${ }^{42}$ M. Plagge, ${ }^{42}$ G. Quast,${ }^{42}$ K. Rabbertz ${ }^{42}$ M. Schröder, ${ }^{42}$ I. Shvetsov, ${ }^{42}$ G. Sieber, ${ }^{42}$ H. J. Simonis, ${ }^{42}$ R. Ulrich, ${ }^{42}$ S. Wayand, ${ }^{42}$ M. Weber, ${ }^{42}$ T. Weiler, ${ }^{42}$ S. Williamson, ${ }^{42}$ C. Wöhrmann, ${ }^{42}$ R. Wolf,${ }^{42}$ G. Anagnostou, ${ }^{43}$ G. Daskalakis, ${ }^{43}$ T. Geralis, ${ }^{43}$ V. A. Giakoumopoulou, ${ }^{43}$ A. Kyriakis, ${ }^{43}$ D. Loukas, ${ }^{43}$ I. Topsis-Giotis, ${ }^{43}$ G. Karathanasis, ${ }^{44}$ S. Kesisoglou, ${ }^{44}$ A. Panagiotou, ${ }^{44}$ N. Saoulidou, ${ }^{44}$ K. Kousouris, ${ }^{45}$ I. Evangelou, ${ }^{46}$ C. Foudas,${ }^{46}$ P. Kokkas, ${ }^{46}$ S. Mallios, ${ }^{46}$ N. Manthos, ${ }^{46}$ I. Papadopoulos ${ }^{46}$ E. Paradas, ${ }^{46}$ J. Strologas ${ }^{46}$ F. A. Triantis, ${ }^{46}$ M. Csanad, ${ }^{47}$ N. Filipovic, ${ }^{47}$ G. Pasztor, ${ }^{47}$ O. Surányi, ${ }^{47}$ G. I. Veres,${ }^{47, t}$ G. Bencze,${ }^{48}$

C. Hajdu, ${ }^{48}$ D. Horvath, ${ }^{48, u}$ Á. Hunyadi, ${ }^{48}$ F. Sikler, ${ }^{48}$ V. Veszpremi, ${ }^{48}$ A. J. Zsigmond, ${ }^{48}$ N. Beni, ${ }^{49}$ S. Czellar, ${ }^{49}$

J. Karancsi, ${ }^{49, v}$ A. Makovec, ${ }^{49}$ J. Molnar, ${ }^{49}$ Z. Szillasi, ${ }^{49}$ M. Bartók, ${ }^{50, t}$ P. Raics,${ }^{50}$ Z. L. Trocsanyi,${ }^{50}$ B. Ujvari, ${ }^{50}$ S. Choudhury, ${ }^{51}$ J. R. Komaragiri, ${ }^{51}$ S. Bahinipati, ${ }^{52, w}$ S. Bhowmik, ${ }^{52}$ P. Mal,${ }^{52}$ K. Mandal ${ }^{52}$ A. Nayak ${ }^{52, x}$ D. K. Sahoo, ${ }^{52, w}$ N. Sahoo, ${ }^{52}$ S. K. Swain, ${ }^{52}$ S. Bansal, ${ }^{53}$ S. B. Beri, ${ }^{53}$ V. Bhatnagar, ${ }^{53}$ R. Chawla, ${ }^{53}$ N. Dhingra, ${ }^{53}$ A. K. Kalsi, ${ }^{53}$ A. Kaur, ${ }^{53}$ M. Kaur ${ }^{53}$ S. Kaur,${ }^{53}$ R. Kumar, ${ }^{53}$ P. Kumari, ${ }^{53}$ A. Mehta, ${ }^{53}$ J. B. Singh ${ }^{53}$ G. Walia, ${ }^{53}$ Ashok Kumar,${ }^{54}$ Aashaq Shah, ${ }^{54}$ A. Bhardwaj, ${ }^{54}$ S. Chauhan, ${ }^{54}$ B. C. Choudhary, ${ }^{54}$ R. B. Garg,${ }^{54}$ S. Keshri, ${ }^{54}$ A. Kumar, ${ }^{54}$ S. Malhotra, ${ }^{54}$ M. Naimuddin, ${ }^{54}$ K. Ranjan, ${ }^{54}$ R. Sharma,${ }^{54}$ R. Bhardwaj, ${ }^{55}$ R. Bhattacharya ${ }^{55}$ S. Bhattacharya,${ }^{55}$ U. Bhawandeep, ${ }^{55}$ S. Dey,${ }^{55}$ S. Dutt, ${ }^{55}$ S. Dutta, ${ }^{55}$ S. Ghosh,${ }^{55}$ N. Majumdar, ${ }^{55}$ A. Modak, ${ }^{55}$ K. Mondal, ${ }^{55}$ S. Mukhopadhyay, ${ }^{55}$ S. Nandan, ${ }^{55}$ A. Purohit, ${ }^{55}$ A. Roy, ${ }^{55}$ D. Roy, ${ }^{55}$ S. Roy Chowdhury ${ }^{55}$ S. Sarkar,${ }^{55}$ M. Sharan ${ }^{55}$ S. Thakur, ${ }^{55}$ P. K. Behera,${ }^{56}$ R. Chudasama, ${ }^{57}$ D. Dutta, ${ }^{57}$ V. Jha,${ }^{57}$

V. Kumar, ${ }^{57}$ A. K. Mohanty, ${ }^{57, p}$ P. K. Netrakanti ${ }^{57}$ L. M. Pant, ${ }^{57}$ P. Shukla, ${ }^{57}$ A. Topkar, ${ }^{57}$ T. Aziz, ${ }^{58}$ S. Dugad, ${ }^{58}$ B. Mahakud ${ }^{58}$ S. Mitra,${ }^{58}$ G. B. Mohanty,${ }^{58}$ N. Sur,${ }^{58}$ B. Sutar, ${ }^{58}$ S. Banerjee,${ }^{59}$ S. Bhattacharya,${ }^{59}$ S. Chatterjee,${ }^{59}$ P. Das,${ }^{59}$ M. Guchait, ${ }^{59}$ Sa. Jain, ${ }^{59}$ S. Kumar, ${ }^{59}$ M. Maity, ${ }^{59, y}$ G. Majumder, ${ }^{59}$ K. Mazumdar, ${ }^{59}$ T. Sarkar, ${ }^{59, y}$ N. Wickramage, ${ }^{59, z}$ S. Chauhan, ${ }^{60}$ S. Dube ${ }^{60}$ V. Hegde ${ }^{60}$ A. Kapoor, ${ }^{60}$ K. Kothekar, ${ }^{60}$ S. Pandey,${ }^{60}$ A. Rane, ${ }^{60}$ S. Sharma, ${ }^{60}$ S. Chenarani, ${ }^{61 \text {,aa }}$ E. Eskandari Tadavani ${ }^{61}$ S. M. Etesami, ${ }^{61, a a}$ M. Khakzad, ${ }^{61}$ M. Mohammadi Najafabadi, ${ }^{61}$ M. Naseri, ${ }^{61}$

S. Paktinat Mehdiabadi, ${ }^{61, b b}$ F. Rezaei Hosseinabadi, ${ }^{61}$ B. Safarzadeh, ${ }^{61, c c}$ M. Zeinali, ${ }^{61}$ M. Felcini, ${ }^{62}$ M. Grunewald, ${ }^{62}$ M. Abbrescia ${ }^{63 a, 63 b}$ C. Calabria, ${ }^{63 a, 63 b}$ A. Colaleo, ${ }^{63 a}$ D. Creanza, ${ }^{63 a, 63 c}$ L. Cristella, ${ }^{63 a, 63 b}$ N. De Filippis, ${ }^{63 a, 63 c}$ M. De Palma, ${ }^{63 a, 63 b}$ F. Errico, ${ }^{63 a, 63 b}$ L. Fiore ${ }^{63 a}$ G. Iaselli, ${ }^{63 a, 63 c}$ S. Lezki, ${ }^{63 a, 63 b}$ G. Maggi, ${ }^{63 a, 63 c}$ M. Maggi, ${ }^{63 a}$ G. Miniello, ${ }^{63 a, 63 b}$ S. My ${ }^{63 a, 63 b}$ S. Nuzzo, ${ }^{63 a, 63 b}$ A. Pompili, ${ }^{63 a, 63 b}$ G. Pugliese,${ }^{63 a, 63 c}$ R. Radogna,${ }^{63 a}$ A. Ranieri, ${ }^{63 a}$ G. Selvaggi, ${ }^{63 a, 63 b}$ A. Sharma, ${ }^{63 a}$ L. Silvestris, ${ }^{63 a, p}$ R. Venditti, ${ }^{63 a}$ P. Verwilligen, ${ }^{63 a}$ G. Abbiendi, ${ }^{64 a}$ C. Battilana, ${ }^{64 a, 64 b}$ D. Bonacorsi, ${ }^{64 a, 64 b}$ L. Borgonovi, ${ }^{64 a, 64 b}$ S. Braibant-Giacomelli, ${ }^{64 a, 64 b}$ R. Campanini, ${ }^{64 a, 64 b}$ P. Capiluppi, ${ }^{64 a, 64 b}$ A. Castro, ${ }^{64 a 64 b}$ F. R. Cavallo, ${ }^{64 a}$ S. S. Chhibra ${ }^{64 a}$ G. Codispoti ${ }^{64 a, 64 b}$ M. Cuffiani,${ }^{64 a, 64 b}$ G. M. Dallavalle ${ }^{64 a}$ F. Fabbri, ${ }^{64 a}$ A. Fanfani, ${ }^{64 a, 64 b}$ D. Fasanella, ${ }^{64 a, 64 b}$ P. Giacomelli, ${ }^{64 a}$ C. Grandi, ${ }^{64 a}$ L. Guiducci, ${ }^{64 a, 64 b}$ S. Marcellini, ${ }^{64 a}$ G. Masetti, ${ }^{64 a}$ A. Montanari, ${ }^{64 \mathrm{a}}$ F. L. Navarria, ${ }^{64 a, 64 b}$ A. Perrotta, ${ }^{64 \mathrm{a}}$ A. M. Rossi, ${ }^{64 a, 64 b}$ T. Rovelli, ${ }^{64,64 b}$ G. P. Siroli, ${ }^{64 a, 64 b}$ N. Tosi, ${ }^{64 a}$ S. Albergo,${ }^{65 a, 65 b}$ S. Costa, ${ }^{65 a, 65 b}$ A. Di Mattia, ${ }^{65 a}$ F. Giordano,${ }^{65 a, 65 b}$ R. Potenza, ${ }^{65 a, 65 b}$ A. Tricomi ${ }^{65 a, 65 b}$ C. Tuve, ${ }^{65 a, 65 b}$ G. Barbagli, ${ }^{66 \mathrm{a}}$ K. Chatterjee ${ }^{66 a, 66 \mathrm{~b}}$ V. Ciulli, ${ }^{66 a, 66 \mathrm{~b}}$ C. Civinini, ${ }^{66 \mathrm{a}}$ R. D’Alessandro, ${ }^{66 a, 66 b}$ E. Focardi, ${ }^{66 a, 66 b}$ P. Lenzi, ${ }^{66 a, 66 b}$ M. Meschini ${ }^{66 a}$ S. Paoletti, ${ }^{66 a}$ L. Russo, ${ }^{66 a, d d}$ G. Sguazzoni, ${ }^{66 a}$ D. Strom, ${ }^{66 a}$ L. Viliani, ${ }^{66 a, 66 b, p}$ L. Benussi ${ }^{67}$ S. Bianco, ${ }^{67}$ F. Fabbri, ${ }^{67}$ D. Piccolo, ${ }^{67}$ F. Primavera, ${ }^{67, p}$ V. Calvelli, ${ }^{68 a, 68 b}$ F. Ferro, ${ }^{68 a}$ E. Robutti ${ }^{68 a}$ S. Tosi ${ }^{68 a, 68 b}$ A. Benaglia, ${ }^{69 a}$ L. Brianza,${ }^{69 a, 69 b}$ F. Brivio, ${ }^{69 a, 69 b}$ V. Ciriolo, ${ }^{69 a, 69 b}$ M. E. Dinardo ${ }^{69 a, 69 b}$ S. Fiorendi, ${ }^{69 a, 69 b}$ S. Gennai, ${ }^{69 a}$ A. Ghezzi, ${ }^{69 a, 69 b}$ P. Govoni, ${ }^{69 a, 69 b}$ M. Malberti, ${ }^{69 a, 69 b}$ S. Malvezzi, ${ }^{69 a}$ R. A. Manzoni, ${ }^{69 a, 69 b}$ D. Menasce, ${ }^{69 a}$ L. Moroni, ${ }^{69 a}$ M. Paganoni, ${ }^{69 a, 69 b}$ K. Pauwels, ${ }^{69 a, 69 b}$ D. Pedrini, ${ }^{69 \mathrm{a}}$ S. Pigazzini, ${ }^{69 \mathrm{a}, 69 \mathrm{~b}, \mathrm{ee}}$ S. Ragazzi, ${ }^{69 a, 69 \mathrm{~b}}$ N. Redaelli, ${ }^{69 \mathrm{a}}$ T. Tabarelli de Fatis ${ }^{69 \mathrm{a}, 69 \mathrm{~b}}$

S. Buontempo, ${ }^{70 \mathrm{a}}$ N. Cavallo, ${ }^{70 \mathrm{a}, 70 \mathrm{c}}$ S. Di Guida,${ }^{70 \mathrm{a}, 70 \mathrm{~d}, \mathrm{p}}$ F. Fabozzi, ${ }^{70 \mathrm{a}, 70 \mathrm{c}}$ F. Fienga, ${ }^{70 \mathrm{a}, 70 \mathrm{~b}}$ A. O. M. Iorio, ${ }^{70 a, 70 \mathrm{~b}}$ W. A. Khan, ${ }^{70 a}$ L. Lista, ${ }^{70 a}$ S. Meola, ${ }^{70 a, 70 d, p}$ P. Paolucci, ${ }^{70 a, p}$ C. Sciacca, ${ }^{70 a, 70 b}$ F. Thyssen, ${ }^{70 a}$ P. Azzi, ${ }^{71 a}$ N. Bacchetta, ${ }^{71 a}$ L. Benato, ${ }^{71 \mathrm{a}, 71 \mathrm{~b}}$ M. Biasotto, ${ }^{71 \mathrm{a}, \mathrm{ff}}$ D. Bisello, ${ }^{71 \mathrm{a}, 71 \mathrm{~b}}$ A. Boletti, ${ }^{71 \mathrm{a}, 71 \mathrm{~b}}$ R. Carlin, ${ }^{71 \mathrm{a}, 71 \mathrm{~b}}$ A. Carvalho Antunes De Oliveira, ${ }^{71 \mathrm{a}, 71 \mathrm{~b}}$ P. Checchia, ${ }^{71 \mathrm{a}}$ M. Dall'Osso, ${ }^{71 \mathrm{a}, 71 \mathrm{~b}}$ P. De Castro Manzano, ${ }^{71 \mathrm{a}}$ T. Dorigo, ${ }^{71 \mathrm{a}}$ U. Gasparini, ${ }^{71,71 \mathrm{~b}}$ A. Gozzelino, ${ }^{71 \mathrm{a}}$ S. Lacaprara, ${ }^{71 \mathrm{a}}$ P. Lujan, ${ }^{71 \mathrm{a}}$ M. Margoni, ${ }^{71 \mathrm{a}, 71 \mathrm{~b}}$ A. T. Meneguzzo, ${ }^{71 \mathrm{a}, 71 \mathrm{~b}}$ N. Pozzobon, ${ }^{71,71 \mathrm{~b}}$ P. Ronchese, ${ }^{71 \mathrm{a}, 71 \mathrm{~b}}$ R. Rossin, ${ }^{71 a, 71 b}$ F. Simonetto, ${ }^{71 a, 71 b}$ E. Torassa, ${ }^{71 a}$ S. Ventura, ${ }^{71 a}$ M. Zanetti, ${ }^{71 a, 71 b}$ P. Zotto, ${ }^{71 a, 71 b}$ A. Braghieri, ${ }^{72 a}$ 
A. Magnani, ${ }^{72 a}$ P. Montagna, ${ }^{72 a, 72 b}$ S. P. Ratti, ${ }^{72 a, 72 b}$ V. Re,${ }^{72 a}$ M. Ressegotti, ${ }^{72 a, 72 b}$ C. Riccardi, ${ }^{72 a, 72 b}$ P. Salvini, ${ }^{72 a}$ I. Vai, ${ }^{72 a, 72 b}$ P. Vitulo ${ }^{72 a, 72 b}$ L. Alunni Solestizi ${ }^{73 a, 73 b}$ M. Biasini, ${ }^{73 a, 73 b}$ G. M. Bilei, ${ }^{73 a}$ C. Cecchi, ${ }^{73 a, 73 b}$ D. Ciangottini, ${ }^{73 a, 73 b}$ L. Fanò ${ }^{73 a, 73 b}$ P. Lariccia,${ }^{73 a, 73 b}$ R. Leonardi, ${ }^{73 a, 73 b}$ E. Manoni ${ }^{73 a}$ G. Mantovani,${ }^{73 a, 73 b}$ V. Mariani, ${ }^{73 a, 73 b}$ M. Menichelli, ${ }^{73 a}$ A. Rossi, ${ }^{73 a, 73 b}$ A. Santocchia, ${ }^{73 a, 73 b}$ D. Spiga ${ }^{73 a}$ K. Androsov, ${ }^{74 a}$ P. Azzurri, ${ }^{74 a, p}$ G. Bagliesi, ${ }^{74 a}$ T. Boccali, ${ }^{74 a}$ L. Borrello, ${ }^{74 a}$ R. Castaldi ${ }^{74 \mathrm{a}}$ M. A. Ciocci, ${ }^{74 a, 74 b}$ R. Dell'Orso, ${ }^{74 a}$ G. Fedi, ${ }^{74 a}$ L. Giannini,${ }^{74 a, 74 c}$ A. Giassi, ${ }^{74 a}$ M. T. Grippo, ${ }^{74, d d}$ F. Ligabue ${ }^{74 a, 74 c}$ T. Lomtadze, ${ }^{74 a}$ E. Manca, ${ }^{74 a, 74 c}$ G. Mandorlii, ${ }^{74 a, 74 c}$ L. Martini, ${ }^{74 a, 74 b}$ A. Messineo ${ }^{74 a, 74 b}$ F. Palla, ${ }^{74 a}$ A. Rizzi,${ }^{74 a}{ }^{74 b}$ A. Savoy-Navarro, ${ }^{74 a, g g}$ P. Spagnolo,${ }^{74 a}$ R. Tenchini, ${ }^{74 a}$ G. Tonelli, ${ }^{74 a}{ }^{74 b}$ A. Venturi, ${ }^{74 a}$ P. G. Verdini, ${ }^{74 a}$ L. Barone,${ }^{75 a, 75 b}$ F. Cavallari, ${ }^{75 a}$ M. Cipriani, ${ }^{75 a, 75 b}$ N. Daci ${ }^{75 a}$ D. Del Re,${ }^{75 a, 75 b, p}$ E. Di Marco ${ }^{75 a, 75 b}$ M. Diemoz, ${ }^{75 a}$ S. Gelli, ${ }^{75 a, 75 b}$ E. Longo, ${ }^{75 a, 75 b}$ F. Margaroli, ${ }^{75 a, 75 b}$ B. Marzocchi, ${ }^{75 a, 75 b}$ P. Meridiani, ${ }^{75 a}$ G. Organtini, ${ }^{75 a, 75 b}$ R. Paramatti, ${ }^{75 a, 75 b}$ F. Preiato, ${ }^{75 a, 75 b}$ S. Rahatlou, ${ }^{75 a, 75 b}$ C. Rovelli, ${ }^{75 a}$ F. Santanastasio, ${ }^{75 a, 75 b}$ N. Amapane, ${ }^{76 a, 76 b}$ R. Arcidiacono, ${ }^{76 a, 76 c}$ S. Argiro, ${ }^{76 a, 76 b}$ M. Arneodo, ${ }^{76 a, 76 c}$ N. Bartosik, ${ }^{76 a}$ R. Bellan, ${ }^{76 a, 76 b}$ C. Biino,${ }^{76 a}$ N. Cartiglia, ${ }^{76 a}$ F. Cenna, ${ }^{76 a, 76 b}$ M. Costa, ${ }^{76 a, 76 b}$ R. Covarelli, ${ }^{76 a, 76 b}$ A. Degano, ${ }^{76 a, 76 b}$ N. Demaria ${ }^{76 a}$ B. Kiani, ${ }^{76 a, 76 b}$ C. Mariotti, ${ }^{76 a}$ S. Maselli, ${ }^{76 a}$ E. Migliore, ${ }^{76 a, 76 b}$ V. Monaco, ${ }^{76 a, 76 b}$ E. Monteil,${ }^{76 a, 76 b}$ M. Monteno, ${ }^{76 a}$ M. M. Obertino, ${ }^{76 a, 76 b}$ L. Pacher,${ }^{76 a, 76 b}$ N. Pastrone, ${ }^{76 a}$

M. Pelliccioni, ${ }^{76 a}$ G. L. Pinna Angioni ${ }^{76 a, 76 b}$ F. Ravera, ${ }^{76 a, 76 b}$ A. Romero, ${ }^{76 a, 76 b}$ M. Ruspa,${ }^{76 a, 76 c}$ R. Sacchi, ${ }^{76 a, 76 b}$ K. Shchelina, ${ }^{76 a, 76 b}$ V. Sola ${ }^{76 a}$ A. Solano, ${ }^{76 a, 76 b}$ A. Staiano, ${ }^{76 a}$ P. Traczyk,${ }^{76 a, 76 b}$ S. Belforte, ${ }^{77 a}$ M. Casarsa,${ }^{77 a}$ F. Cossutti, ${ }^{77 a}$ G. Della Ricca, ${ }^{77 a, 77 b}$ A. Zanetti, ${ }^{77 a}$ D. H. Kim, ${ }^{78}$ G. N. Kim,${ }^{78}$ M. S. Kim,${ }^{78}$ J. Lee, ${ }^{78}$ S. Lee, ${ }^{78}$ S. W. Lee, ${ }^{78}$ C. S. Moon, ${ }^{78}$ Y. D. Oh ${ }^{78}$ S. Sekmen, ${ }^{78}$ D. C. Son, ${ }^{78}$ Y. C. Yang,${ }^{78}$ A. Lee, ${ }^{79}$ H. Kim,${ }^{80}$ D. H. Moon,${ }^{80}$ G. Oh,${ }^{80}$ J. A. Brochero Cifuentes,${ }^{81}$ J. Goh, ${ }^{81}$ T. J. Kim, ${ }^{81}$ S. Cho, ${ }^{82}$ S. Choi ${ }^{82}$ Y. Go, ${ }^{82}$ D. Gyun, ${ }^{82}$ S. Ha, ${ }^{82}$ B. Hong, ${ }^{82}$ Y. Jo, ${ }^{82}$ Y. Kim ${ }^{82}$ K. Lee, ${ }^{82}$ K. S. Lee, ${ }^{82}$ S. Lee, ${ }^{82}$ J. Lim, ${ }^{82}$ S. K. Park, ${ }^{82}$ Y. Roh,${ }^{82}$ J. Almond, ${ }^{83}$ J. Kim, ${ }^{83}$ J. S. Kim, ${ }^{83}$ H. Lee, ${ }^{83}$ K. Lee, ${ }^{83}$ K. Nam ${ }^{83}$ S. B. Oh, ${ }^{83}$ B. C. Radburn-Smith, ${ }^{83}$ S. h. Seo ${ }^{83}$ U. K. Yang, ${ }^{83}$ H. D. Yoo, ${ }^{83}$ G. B. Yu, ${ }^{83}$ M. Choi, ${ }^{84}$ H. Kim, ${ }^{84}$ J. H. Kim,${ }^{84}$ J. S. H. Lee, ${ }^{84}$ I. C. Park, ${ }^{84}$ Y. Choi ${ }^{85}$ C. Hwang, ${ }^{85}$ J. Lee, ${ }^{85}$ I. Yu, ${ }^{85}$ V. Dudenas, ${ }^{86}$ A. Juodagalvis, ${ }^{86}$ J. Vaitkus, ${ }^{86}$ I. Ahmed, ${ }^{87}$ Z. A. Ibrahim, ${ }^{87}$ M. A. B. Md Ali, ${ }^{87, h h}$ F. Mohamad Idris, ${ }^{87, \text { ii }}$ W. A. T. Wan Abdullah, ${ }^{87}$ M. N. Yusli, ${ }^{87}$ Z. Zolkapli, ${ }^{87}$ R. Reyes-Almanza, ${ }^{88}$ G. Ramirez-Sanchez, ${ }^{88}$ M. C. Duran-Osuna, ${ }^{88}$ H. Castilla-Valdez, ${ }^{88}$ E. De La Cruz-Burelo, ${ }^{88}$ I. Heredia-De La Cruz, ${ }^{88, j j}$ R. I. Rabadan-Trejo, ${ }^{88}$ R. Lopez-Fernandez, ${ }^{88}$ J. Mejia Guisao, ${ }^{88}$ A. Sanchez-Hernandez, ${ }^{88}$ S. Carrillo Moreno, ${ }^{89}$ C. Oropeza Barrera, ${ }^{89}$ F. Vazquez Valencia, ${ }^{89}$ I. Pedraza ${ }^{90}$ H. A. Salazar Ibarguen, ${ }^{90}$

C. Uribe Estrada, ${ }^{90}$ A. Morelos Pineda, ${ }^{91}$ D. Krofcheck, ${ }^{92}$ P. H. Butler, ${ }^{93}$ A. Ahmad ${ }^{94}$ M. Ahmad, ${ }^{94}$ Q. Hassan, ${ }^{94}$ H. R. Hoorani, ${ }^{94}$ A. Saddique, ${ }^{94}$ M. A. Shah, ${ }^{94}$ M. Shoaib, ${ }^{94}$ M. Waqas, ${ }^{94}$ H. Bialkowska, ${ }^{95}$ M. Bluj,${ }^{95}$ B. Boimska ${ }^{95}$ T. Frueboes, ${ }^{95}$ M. Górski, ${ }^{95}$ M. Kazana, ${ }^{95}$ K. Nawrocki,${ }^{95}$ M. Szleper,${ }^{95}$ P. Zalewski, ${ }^{95}$ K. Bunkowski, ${ }^{96}$ A. Byszuk, ${ }^{96, k k}$ K. Doroba, ${ }^{96}$ A. Kalinowski, ${ }^{96}$ M. Konecki, ${ }^{96}$ J. Krolikowski, ${ }^{96}$ M. Misiura, ${ }^{96}$ M. Olszewski, ${ }^{96}$ A. Pyskir, ${ }^{96}$ M. Walczak, ${ }^{96}$ P. Bargassa, ${ }^{97}$ C. Beirão Da Cruz E Silva, ${ }^{97}$ A. Di Francesco, ${ }^{97}$ P. Faccioli, ${ }^{97}$ B. Galinhas,${ }^{97}$ M. Gallinaro, ${ }^{97}$ J. Hollar, ${ }^{97}$ N. Leonardo, ${ }^{97}$ L. Lloret Iglesias, ${ }^{97}$ M. V. Nemallapudi, ${ }^{97}$ J. Seixas, ${ }^{97}$ G. Strong, ${ }^{97}$ O. Toldaiev,${ }^{97}$ D. Vadruccio, ${ }^{97}$ J. Varela, ${ }^{97}$ S. Afanasiev, ${ }^{98}$ P. Bunin, ${ }^{98}$ M. Gavrilenko, ${ }^{98}$ I. Golutvin, ${ }^{98}$ I. Gorbunov, ${ }^{98}$ A. Kamenev ${ }^{98}$ V. Karjavin, ${ }^{98}$ A. Lanev, ${ }^{98}$ A. Malakhov, ${ }^{98}$ V. Matveev, ${ }^{98,11, m m}$ V. Palichik, ${ }^{98}$ V. Perelygin, ${ }^{98}$ S. Shmatov, ${ }^{98}$ S. Shulha,${ }^{98}$ N. Skatchkov,${ }^{98}$ V. Smirnov,${ }^{98}$ N. Voytishin, ${ }^{98}$ A. Zarubin, ${ }^{98}$ Y. Ivanov, ${ }^{99}$ V. Kim, ${ }^{99, n n}$ E. Kuznetsova, ${ }^{99, \text { oo }}$ P. Levchenko, ${ }^{99}$ V. Murzin, ${ }^{99}$ V. Oreshkin,,${ }^{99}$ I. Smirnov, ${ }^{99}$ V. Sulimov, ${ }^{99}$ L. Uvarov,${ }^{99}$ S. Vavilov, ${ }^{99}$ A. Vorobyev,${ }^{99}$ Yu. Andreev, ${ }^{100}$ A. Dermenev, ${ }^{100}$ S. Gninenko, ${ }^{100}$ N. Golubev, ${ }^{100}$ A. Karneyeu, ${ }^{100}$ M. Kirsanov, ${ }^{100}$ N. Krasnikov, ${ }^{100}$ A. Pashenkov, ${ }^{100}$ D. Tlisov, ${ }^{100}$ A. Toropin, ${ }^{100}$ V. Epshteyn, ${ }^{101}$ V. Gavrilov, ${ }^{101}$ N. Lychkovskaya, ${ }^{101}$ V. Popov ${ }^{101}$ I. Pozdnyakov, ${ }^{101}$ G. Safronov, ${ }^{101}$ A. Spiridonov, ${ }^{101}$ A. Stepennov, ${ }^{101}$ M. Toms, ${ }^{101}$ E. Vlasov, ${ }^{101}$ A. Zhokin, ${ }^{101}$ T. Aushev ${ }^{102}$ A. Bylinkin, ${ }^{102, m m}$ R. Chistov, ${ }^{103, p p}$ M. Danilov, ${ }^{103, p p}$ P. Parygin, ${ }^{103}$ D. Philippov, ${ }^{103}$ S. Polikarpov, ${ }^{103}$ E. Tarkovskii, ${ }^{103}$ V. Andreev, ${ }^{104}$ M. Azarkin, ${ }^{104, m m}$ I. Dremin,${ }^{104, m m}$ M. Kirakosyan, ${ }^{104, \mathrm{~mm}}$ A. Terkulov, ${ }^{104}$ A. Baskakov, ${ }^{105}$ A. Belyaev, ${ }^{105}$ E. Boos,,${ }^{105}$ M. Dubinin, ${ }^{105, q q}$ L. Dudko, ${ }^{105}$ A. Ershov, ${ }^{105}$ A. Gribushin, ${ }^{105}$ V. Klyukhin, ${ }^{105}$ O. Kodolova, ${ }^{105}$ I. Lokhtin, ${ }^{105}$ I. Miagkov, ${ }^{105}$ S. Obraztsov, ${ }^{105}$ S. Petrushanko, ${ }^{105}$ V. Savrin, ${ }^{105}$ A. Snigirev, ${ }^{105}$ V. Blinov, ${ }^{106, \text { rr }}$ Y. Skovpen, ${ }^{106, r \mathrm{rr}}$ D. Shtol, ${ }^{106, \mathrm{rr}}$ I. Azhgirey, ${ }^{107}$ I. Bayshev, ${ }^{107}$ S. Bitioukov, ${ }^{107}$ D. Elumakhov, ${ }^{107}$ V. Kachanov, ${ }^{107}$ A. Kalinin, ${ }^{107}$ D. Konstantinov, ${ }^{107}$ P. Mandrik, ${ }^{107}$ V. Petrov,${ }^{107}$ R. Ryutin, ${ }^{107}$ A. Sobol, ${ }^{107}$ S. Troshin, ${ }^{107}$ N. Tyurin, ${ }^{107}$ A. Uzunian, ${ }^{107}$ A. Volkov,${ }^{107}$ P. Adzic, ${ }^{108, s s}$ P. Cirkovic, ${ }^{108}$ D. Devetak, ${ }^{108}$ M. Dordevic, ${ }^{108}$ J. Milosevic, ${ }^{108}$ V. Rekovic, ${ }^{108}$ J. Alcaraz Maestre, ${ }^{109}$ M. Barrio Luna,${ }^{109}$ M. Cerrada, ${ }^{109}$ N. Colino, ${ }^{109}$ B. De La Cruz, ${ }^{109}$ A. Delgado Peris, ${ }^{109}$ A. Escalante Del Valle, ${ }^{109}$ C. Fernandez Bedoya,${ }^{109}$

J. P. Fernández Ramos, ${ }^{109}$ J. Flix, ${ }^{109}$ M. C. Fouz, ${ }^{109}$ P. Garcia-Abia, ${ }^{109}$ O. Gonzalez Lopez, ${ }^{109}$ S. Goy Lopez, ${ }^{109}$ J. M. Hernandez, ${ }^{109}$ M. I. Josa, ${ }^{109}$ D. Moran ${ }^{109}$ A. Pérez-Calero Yzquierdo, ${ }^{109}$ J. Puerta Pelayo, ${ }^{109}$ A. Quintario Olmeda, ${ }^{109}$ I. Redondo, ${ }^{109}$ L. Romero, ${ }^{109}$ M. S. Soares, ${ }^{109}$ A. Álvarez Fernández, ${ }^{109}$ C. Albajar, ${ }^{110}$ J. F. de Trocóniz, ${ }^{110}$ M. Missiroli, ${ }^{110}$ 
J. Cuevas, ${ }^{111}$ C. Erice, ${ }^{111}$ J. Fernandez Menendez, ${ }^{111}$ I. Gonzalez Caballero, ${ }^{111}$ J. R. González Fernández, ${ }^{111}$ E. Palencia Cortezon, ${ }^{111}$ S. Sanchez Cruz, ${ }^{111}$ P. Vischia, ${ }^{111}$ J. M. Vizan Garcia, ${ }^{111}$ I. J. Cabrillo, ${ }^{112}$ A. Calderon, ${ }^{112}$ B. Chazin Quero, ${ }^{112}$ E. Curras, ${ }^{112}$ J. Duarte Campderros, ${ }^{112}$ M. Fernandez, ${ }^{112}$ J. Garcia-Ferrero, ${ }^{112}$ G. Gomez, ${ }^{112}$ A. Lopez Virto, ${ }^{112}$ J. Marco, ${ }^{112}$ C. Martinez Rivero, ${ }^{112}$ P. Martinez Ruiz del Arbol, ${ }^{112}$ F. Matorras, ${ }^{112}$ J. Piedra Gomez, ${ }^{112}$ T. Rodrigo, ${ }^{112}$ A. Ruiz-Jimeno, ${ }^{112}$ L. Scodellaro, ${ }^{112}$ N. Trevisani, ${ }^{112}$ I. Vila, ${ }^{112}$ R. Vilar Cortabitarte, ${ }^{112}$ D. Abbaneo, ${ }^{113}$ B. Akgun, ${ }^{113}$ E. Auffray, ${ }^{113}$ P. Baillon, ${ }^{113}$ A. H. Ball ${ }^{113}$ D. Barney, ${ }^{113}$ M. Bianco, ${ }^{113}$ P. Bloch, ${ }^{113}$ A. Bocci, ${ }^{113}$ C. Botta, ${ }^{113}$

T. Camporesi, ${ }^{113}$ R. Castello, ${ }^{113}$ M. Cepeda, ${ }^{113}$ G. Cerminara, ${ }^{113}$ E. Chapon, ${ }^{113}$ Y. Chen, ${ }^{113}$ D. d'Enterria, ${ }^{113}$ A. Dabrowski, ${ }^{113}$ V. Daponte, ${ }^{113}$ A. David, ${ }^{113}$ M. De Gruttola, ${ }^{113}$ A. De Roeck, ${ }^{113}$ N. Deelen, ${ }^{113}$ M. Dobson, ${ }^{113}$ T. du Pree, ${ }^{113}$ M. Dünser, ${ }^{113}$ N. Dupont, ${ }^{113}$ A. Elliott-Peisert, ${ }^{113}$ P. Everaerts, ${ }^{113}$ F. Fallavollita, ${ }^{113}$ G. Franzoni, ${ }^{113}$ J. Fulcher, ${ }^{113}$ W. Funk, ${ }^{113}$ D. Gigi, ${ }^{113}$ A. Gilbert, ${ }^{113}$ K. Gill, ${ }^{113}$ F. Glege, ${ }^{113}$ D. Gulhan, ${ }^{113}$ P. Harris, ${ }^{113}$ J. Hegeman, ${ }^{113}$ V. Innocente, ${ }^{113}$ A. Jafari, ${ }^{113}$ P. Janot, ${ }^{113}$ O. Karacheban, ${ }^{11, s}$ J. Kieseler, ${ }^{113}$ V. Knünz, ${ }^{113}$ A. Kornmayer, ${ }^{113}$ M. J. Kortelainen, ${ }^{113}$ M. Krammer, ${ }^{13, b}$ C. Lange, ${ }^{113}$ P. Lecoq, ${ }^{113}$ C. Lourenço, ${ }^{113}$ M. T. Lucchini, ${ }^{113}$ L. Malgeri, ${ }^{113}$ M. Mannelli, ${ }^{113}$ A. Martelli, ${ }^{113}$ F. Meijers, ${ }^{113}$ J. A. Merlin, ${ }^{113}$ S. Mersi, ${ }^{113}$ E. Meschi, ${ }^{113}$ P. Milenovic, ${ }^{113, t \mathrm{tt}}$ F. Moortgat,${ }^{113}$ M. Mulders ${ }^{113}$ H. Neugebauer, ${ }^{113}$ J. Ngadiuba, ${ }^{113}$ S. Orfanelli, ${ }^{113}$ L. Orsini, ${ }^{113}$ L. Pape,${ }^{113}$ E. Perez,${ }^{113}$ M. Peruzzi, ${ }^{113}$ A. Petrilli, ${ }^{113}$ G. Petrucciani, ${ }^{113}$ A. Pfeiffer, ${ }^{113}$ M. Pierini, ${ }^{113}$ D. Rabady, ${ }^{113}$ A. Racz, ${ }^{113}$ T. Reis, ${ }^{113}$ G. Rolandi, ${ }^{113, \text { uu }}$ M. Rovere,${ }^{113}$ H. Sakulin, ${ }^{113}$ C. Schäfer, ${ }^{113}$ C. Schwick, ${ }^{113}$ M. Seidel,${ }^{113}$ M. Selvaggi, ${ }^{113}$ A. Sharma, ${ }^{113}$ P. Silva,${ }^{113}$ P. Sphicas, ${ }^{113, v v}$ A. Stakia, ${ }^{113}$ J. Steggemann, ${ }^{113}$ M. Stoye,${ }^{113}$ M. Tosi, ${ }^{113}$ D. Treille, ${ }^{113}$ A. Triossi, ${ }^{113}$ A. Tsirou ${ }^{113}$ V. Veckalns, ${ }^{113, w w}$ M. Verweij ${ }^{113}$ W. D. Zeuner, ${ }^{113}$ W. Bertl, ${ }^{114, a}$ L. Caminada, ${ }^{114, x x}$ K. Deiters,${ }^{114}$ W. Erdmann, ${ }^{114}$ R. Horisberger, ${ }^{114}$ Q. Ingram, ${ }^{114}$ H. C. Kaestli, ${ }^{114}$ D. Kotlinski, ${ }^{114}$ U. Langenegger, ${ }^{114}$ T. Rohe, ${ }^{114}$ S. A. Wiederkehr, ${ }^{114}$ M. Backhaus, ${ }^{115}$ L. Bäni, ${ }^{115}$ P. Berger ${ }^{115}$ L. Bianchini, ${ }^{115}$ B. Casal, ${ }^{115}$ G. Dissertori, ${ }^{115}$ M. Dittmar, ${ }^{115}$ M. Donegà, ${ }^{115}$ C. Dorfer, ${ }^{115}$ C. Grab, ${ }^{115}$ C. Heidegger, ${ }^{115}$ D. Hits, ${ }^{115}$ J. Hoss, ${ }^{115}$ G. Kasieczka, ${ }^{115}$ T. Klijnsma, ${ }^{115}$ W. Lustermann, ${ }^{115}$ B. Mangano, ${ }^{115}$

M. Marionneau, ${ }^{115}$ M. T. Meinhard, ${ }^{115}$ D. Meister, ${ }^{115}$ F. Micheli, ${ }^{115}$ P. Musella, ${ }^{115}$ F. Nessi-Tedaldi, ${ }^{115}$ F. Pandolfi, ${ }^{115}$

J. Pata, ${ }^{115}$ F. Pauss, ${ }^{115}$ G. Perrin, ${ }^{115}$ L. Perrozzi, ${ }^{115}$ M. Quittnat, ${ }^{115}$ M. Reichmann, ${ }^{115}$ D. A. Sanz Becerra, ${ }^{115}$ M. Schönenberger ${ }^{115}$ L. Shchutska, ${ }^{115}$ V. R. Tavolaro, ${ }^{115}$ K. Theofilatos,${ }^{115}$ M. L. Vesterbacka Olsson, ${ }^{115}$ R. Wallny, ${ }^{115}$ D. H. Zhu, ${ }^{115}$ T. K. Aarrestad, ${ }^{116}$ C. Amsler, ${ }^{116, y y}$ M. F. Canelli, ${ }^{116}$ A. De Cosa,${ }^{116}$ R. Del Burgo, ${ }^{116}$ S. Donato, ${ }^{116}$ C. Galloni, ${ }^{116}$ T. Hreus, ${ }^{116}$ B. Kilminster, ${ }^{116}$ D. Pinna, ${ }^{116}$ G. Rauco, ${ }^{116}$ P. Robmann, ${ }^{116}$ D. Salerno, ${ }^{116}$ K. Schweiger, ${ }^{116}$ C. Seitz,${ }^{116}$ Y. Takahashi, ${ }^{116}$ A. Zucchetta, ${ }^{116}$ V. Candelise, ${ }^{117}$ T. H. Doan,${ }^{117}$ Sh. Jain, ${ }^{117}$ R. Khurana, ${ }^{117}$ C. M. Kuo, ${ }^{117}$ W. Lin, ${ }^{117}$ A. Pozdnyakov, ${ }^{117}$ S. S. Yu, ${ }^{117}$ Arun Kumar, ${ }^{118}$ P. Chang, ${ }^{118}$ Y. Chao, ${ }^{118}$ K. F. Chen,${ }^{118}$ P. H. Chen,${ }^{118}$ F. Fiori, ${ }^{118}$

W.-S. Hou, ${ }^{118}$ Y. Hsiung, ${ }^{118}$ Y. F. Liu, ${ }^{118}$ R.-S. Lu, ${ }^{118}$ E. Paganis, ${ }^{118}$ A. Psallidas, ${ }^{118}$ A. Steen, ${ }^{118}$ J. f. Tsai, ${ }^{118}$

B. Asavapibhop, ${ }^{119}$ K. Kovitanggoon, ${ }^{119}$ G. Singh, ${ }^{119}$ N. Srimanobhas, ${ }^{119}$ F. Boran, ${ }^{120}$ S. Cerci, ${ }^{120, z z}$ S. Damarseckin, ${ }^{120}$ Z. S. Demiroglu, ${ }^{120}$ C. Dozen, ${ }^{120}$ I. Dumanoglu, ${ }^{120}$ S. Girgis, ${ }^{120}$ G. Gokbulut, ${ }^{120}$ Y. Guler, ${ }^{120}$ I. Hos, ${ }^{120 \text {,aaa }}$ E. E. Kangal, ${ }^{120, b b b}$ O. Kara, ${ }^{120}$ A. Kayis Topaksu, ${ }^{120}$ U. Kiminsu, ${ }^{120}$ M. Oglakci, ${ }^{120}$ G. Onengut, ${ }^{120, c c c}$ K. Ozdemir, ${ }^{120, d d d}$ D. Sunar Cerci, ${ }^{120, z z}$

B. Tali, ${ }^{120, z z}$ S. Turkcapar, ${ }^{120}$ I. S. Zorbakir, ${ }^{120}$ C. Zorbilmez, ${ }^{120}$ B. Bilin, ${ }^{121}$ G. Karapinar, ${ }^{121, \text { eee }}$ K. Ocalan, ${ }^{121, f f f}$ M. Yalvac, ${ }^{121}$ M. Zeyrek, ${ }^{121}$ E. Gülmez, ${ }^{122}$ M. Kaya, ${ }^{122, g g g}$ O. Kaya,${ }^{122, \text { hhh }}$ S. Tekten, ${ }^{122}$ E. A. Yetkin, ${ }^{122, i i i}$ M. N. Agaras, ${ }^{123}$ S. Atay, ${ }^{123}$ A. Cakir, ${ }^{123}$ K. Cankocak, ${ }^{123}$ B. Grynyov, ${ }^{124}$ L. Levchuk, ${ }^{125}$ F. Ball, ${ }^{126}$ L. Beck, ${ }^{126}$ J. J. Brooke, ${ }^{126}$ D. Burns, ${ }^{126}$ E. Clement, ${ }^{126}$ D. Cussans, ${ }^{126}$ O. Davignon, ${ }^{126}$ H. Flacher, ${ }^{126}$ J. Goldstein, ${ }^{126}$ G. P. Heath, ${ }^{126}$ H. F. Heath, ${ }^{126}$ J. Jacob, ${ }^{126}$ L. Kreczko, ${ }^{126}$ D. M. Newbold, ${ }^{126, j j j}$ S. Paramesvaran, ${ }^{126}$ T. Sakuma, ${ }^{126}$ S. Seif El Nasr-storey, ${ }^{126}$ D. Smith, ${ }^{126}$ V. J. Smith, ${ }^{126}$

K. W. Bell, ${ }^{127}$ A. Belyaev, ${ }^{127, k k k}$ C. Brew,${ }^{127}$ R. M. Brown, ${ }^{127}$ L. Calligaris, ${ }^{127}$ D. Cieri, ${ }^{127}$ D. J. A. Cockerill, ${ }^{127}$

J. A. Coughlan, ${ }^{127}$ K. Harder ${ }^{127}$ S. Harper, ${ }^{127}$ E. Olaiya, ${ }^{127}$ D. Petyt, ${ }^{127}$ C. H. Shepherd-Themistocleous, ${ }^{127}$ A. Thea, ${ }^{127}$

I. R. Tomalin, ${ }^{127}$ T. Williams, ${ }^{127}$ G. Auzinger, ${ }^{128}$ R. Bainbridge, ${ }^{128}$ J. Borg,${ }^{128}$ S. Breeze, ${ }^{128}$ O. Buchmuller, ${ }^{128}$

A. Bundock, ${ }^{128}$ S. Casasso, ${ }^{128}$ M. Citron, ${ }^{128}$ D. Colling, ${ }^{128}$ L. Corpe,${ }^{128}$ P. Dauncey, ${ }^{128}$ G. Davies, ${ }^{128}$ A. De Wit, ${ }^{128}$ M. Della Negra, ${ }^{128}$ R. Di Maria, ${ }^{128}$ A. Elwood, ${ }^{128}$ Y. Haddad, ${ }^{128}$ G. Hall, ${ }^{128}$ G. Iles, ${ }^{128}$ T. James, ${ }^{128}$ R. Lane, ${ }^{128}$ C. Laner, ${ }^{128}$ L. Lyons, ${ }^{128}$ A.-M. Magnan, ${ }^{128}$ S. Malik, ${ }^{128}$ L. Mastrolorenzo, ${ }^{128}$ T. Matsushita, ${ }^{128}$ J. Nash, ${ }^{128}$ A. Nikitenko, ${ }^{128, h}$ V. Palladino, ${ }^{128}$ M. Pesaresi, ${ }^{128}$ D. M. Raymond, ${ }^{128}$ A. Richards, ${ }^{128}$ A. Rose, ${ }^{128}$ E. Scott, ${ }^{128}$ C. Seez, ${ }^{128}$ A. Shtipliyski, ${ }^{128}$ S. Summers, ${ }^{128}$ A. Tapper, ${ }^{128}$ K. Uchida ${ }^{128}$ M. Vazquez Acosta, ${ }^{128,111}$ T. Virdee, ${ }^{128, p}$ N. Wardle, ${ }^{128}$ D. Winterbottom, ${ }^{128}$ J. Wright, ${ }^{128}$ S. C. Zenz, ${ }^{128}$ J. E. Cole, ${ }^{129}$ P. R. Hobson, ${ }^{129}$ A. Khan, ${ }^{129}$ P. Kyberd, ${ }^{129}$ I. D. Reid, ${ }^{129}$ P. Symonds, ${ }^{129}$ L. Teodorescu, ${ }^{129}$ M. Turner, ${ }^{129}$ S. Zahid, ${ }^{129}$ A. Borzou, ${ }^{130}$ K. Call, ${ }^{130}$ J. Dittmann, ${ }^{130}$ K. Hatakeyama ${ }^{130}$ H. Liu, ${ }^{130}$ N. Pastika, ${ }^{130}$ C. Smith,${ }^{130}$ R. Bartek, ${ }^{131}$ A. Dominguez, ${ }^{131}$ A. Buccilli, ${ }^{132}$ S. I. Cooper, ${ }^{132}$ C. Henderson, ${ }^{132}$ P. Rumerio, ${ }^{132}$ C. West, ${ }^{132}$ D. Arcaro, ${ }^{133}$ A. Avetisyan, ${ }^{133}$ T. Bose, ${ }^{133}$ D. Gastler, ${ }^{133}$ D. Rankin, ${ }^{133}$ C. Richardson, ${ }^{133}$ J. Rohlf, ${ }^{133}$ L. Sulak, ${ }^{133}$ 
D. Zou, ${ }^{133}$ G. Benelli, ${ }^{134}$ D. Cutts, ${ }^{134}$ A. Garabedian, ${ }^{134}$ M. Hadley, ${ }^{134}$ J. Hakala, ${ }^{134}$ U. Heintz, ${ }^{134}$ J. M. Hogan, ${ }^{134}$ K. H. M. Kwok, ${ }^{134}$ E. Laird, ${ }^{134}$ G. Landsberg, ${ }^{134}$ J. Lee, ${ }^{134}$ Z. Mao, ${ }^{134}$ M. Narain, ${ }^{134}$ J. Pazzini, ${ }^{134}$ S. Piperov, ${ }^{134}$ S. Sagir, ${ }^{134}$ R. Syarif, ${ }^{134}$ D. Yu, ${ }^{134}$ R. Band, ${ }^{135}$ C. Brainerd, ${ }^{135}$ D. Burns, ${ }^{135}$ M. Calderon De La Barca Sanchez, ${ }^{135}$ M. Chertok, ${ }^{135}$ J. Conway, ${ }^{135}$ R. Conway, ${ }^{135}$ P. T. Cox, ${ }^{135}$ R. Erbacher, ${ }^{135}$ C. Flores, ${ }^{135}$ G. Funk, ${ }^{135}$ M. Gardner, ${ }^{135}$ W. Ko, ${ }^{135}$ R. Lander, ${ }^{135}$ C. Mclean, ${ }^{135}$ M. Mulhearn, ${ }^{135}$ D. Pellett, ${ }^{135}$ J. Pilot, ${ }^{135}$ S. Shalhout, ${ }^{135}$ M. Shi, ${ }^{135}$ J. Smith, ${ }^{135}$ D. Stolp, ${ }^{135}$ K. Tos, ${ }^{135}$ M. Tripathi, ${ }^{135}$ Z. Wang, ${ }^{135}$ M. Bachtis, ${ }^{136}$ C. Bravo, ${ }^{136}$ R. Cousins, ${ }^{136}$ A. Dasgupta, ${ }^{136}$ A. Florent, ${ }^{136}$ J. Hauser, ${ }^{136}$ M. Ignatenko, ${ }^{136}$ N. Mccoll, ${ }^{136}$ S. Regnard, ${ }^{136}$ D. Saltzberg, ${ }^{136}$ C. Schnaible, ${ }^{136}$ V. Valuev, ${ }^{136}$ E. Bouvier, ${ }^{137}$ K. Burt, ${ }^{137}$

R. Clare, ${ }^{137}$ J. Ellison, ${ }^{137}$ J. W. Gary, ${ }^{137}$ S. M. A. Ghiasi Shirazi, ${ }^{137}$ G. Hanson, ${ }^{137}$ J. Heilman, ${ }^{137}$ E. Kennedy, ${ }^{137}$ F. Lacroix, ${ }^{137}$ O. R. Long, ${ }^{137}$ M. Olmedo Negrete, ${ }^{137}$ M. I. Paneva, ${ }^{137}$ W. Si ${ }^{137}$ L. Wang,${ }^{137}$ H. Wei, ${ }^{137}$ S. Wimpenny, ${ }^{137}$ B. R. Yates, ${ }^{137}$ J. G. Branson, ${ }^{138}$ S. Cittolin, ${ }^{138}$ M. Derdzinski, ${ }^{138}$ R. Gerosa, ${ }^{138}$ D. Gilbert, ${ }^{138}$ B. Hashemi, ${ }^{138}$ A. Holzner, ${ }^{138}$ D. Klein, ${ }^{138}$ G. Kole, ${ }^{138}$ V. Krutelyov, ${ }^{138}$ J. Letts, ${ }^{138}$ I. Macneill,${ }^{138}$ M. Masciovecchio, ${ }^{138}$ D. Olivito, ${ }^{138}$ S. Padhi, ${ }^{138}$ M. Pieri, ${ }^{138}$ M. Sani, ${ }^{138}$ V. Sharma ${ }^{138}$ S. Simon, ${ }^{138}$ M. Tadel, ${ }^{138}$ A. Vartak,${ }^{138}$ S. Wasserbaech, ${ }^{138, m m m}$ J. Wood, ${ }^{138}$ F. Würthwein, ${ }^{138}$ A. Yagil, ${ }^{138}$ G. Zevi Della Porta ${ }^{138}$ N. Amin, ${ }^{139}$ R. Bhandari,${ }^{139}$ J. Bradmiller-Feld, ${ }^{139}$ C. Campagnari, ${ }^{139}$ A. Dishaw, ${ }^{139}$ V. Dutta, ${ }^{139}$ M. Franco Sevilla, ${ }^{139}$ C. George, ${ }^{139}$ F. Golf, ${ }^{139}$ L. Gouskos, ${ }^{139}$ J. Gran, ${ }^{139}$ R. Heller, ${ }^{139}$ J. Incandela, ${ }^{139}$ S. D. Mullin, ${ }^{139}$ A. Ovcharova, ${ }^{139}$ H. Qu, ${ }^{139}$ J. Richman, ${ }^{139}$ D. Stuart, ${ }^{139}$ I. Suarez, ${ }^{139}$ J. Yoo, ${ }^{139}$

D. Anderson, ${ }^{140}$ J. Bendavid, ${ }^{140}$ A. Bornheim, ${ }^{140}$ J. M. Lawhorn, ${ }^{140}$ H. B. Newman, ${ }^{140}$ T. Nguyen, ${ }^{140}$ C. Pena, ${ }^{140}$ M. Spiropulu, ${ }^{140}$ J. R. Vlimant, ${ }^{140}$ S. Xie, ${ }^{140}$ Z. Zhang, ${ }^{140}$ R. Y. Zhu, ${ }^{140}$ M. B. Andrews, ${ }^{141}$ T. Ferguson, ${ }^{141}$ T. Mudholkar, ${ }^{141}$ M. Paulini, ${ }^{141}$ J. Russ, ${ }^{141}$ M. Sun,${ }^{141}$ H. Vogel,${ }^{141}$ I. Vorobiev, ${ }^{141}$ M. Weinberg, ${ }^{141}$ J. P. Cumalat, ${ }^{142}$ W. T. Ford, ${ }^{142}$

F. Jensen, ${ }^{142}$ A. Johnson, ${ }^{142}$ M. Krohn, ${ }^{142}$ S. Leontsinis, ${ }^{142}$ T. Mulholland, ${ }^{142}$ K. Stenson, ${ }^{142}$ S. R. Wagner, ${ }^{142}$ J. Alexander, ${ }^{143}$ J. Chaves, ${ }^{143}$ J. Chu, ${ }^{143}$ S. Dittmer, ${ }^{143}$ K. Mcdermott,${ }^{143}$ N. Mirman, ${ }^{143}$ J. R. Patterson, ${ }^{143}$ D. Quach, ${ }^{143}$ A. Rinkevicius, ${ }^{143}$ A. Ryd, ${ }^{143}$ L. Skinnari, ${ }^{143}$ L. Soffi, ${ }^{143}$ S. M. Tan, ${ }^{143}$ Z. Tao, ${ }^{143}$ J. Thom, ${ }^{143}$ J. Tucker, ${ }^{143}$ P. Wittich, ${ }^{143}$ M. Zientek, ${ }^{143}$ S. Abdullin, ${ }^{144}$ M. Albrow, ${ }^{144}$ M. Alyari, ${ }^{144}$ G. Apollinari, ${ }^{144}$ A. Apresyan, ${ }^{144}$ A. Apyan, ${ }^{144}$ S. Banerjee, ${ }^{144}$ L. A. T. Bauerdick, ${ }^{144}$ A. Beretvas, ${ }^{144}$ J. Berryhill, ${ }^{144}$ P. C. Bhat, ${ }^{144}$ G. Bolla,${ }^{144, a}$ K. Burkett, ${ }^{144}$ J. N. Butler, ${ }^{144}$ A. Canepa, ${ }^{144}$ G. B. Cerati, ${ }^{144}$ H. W. K. Cheung, ${ }^{144}$ F. Chlebana, ${ }^{144}$ M. Cremonesi, ${ }^{144}$ J. Duarte, ${ }^{144}$ V. D. Elvira, ${ }^{144}$ J. Freeman, ${ }^{144}$ Z. Gecse, ${ }^{144}$ E. Gottschalk, ${ }^{144}$ L. Gray, ${ }^{144}$ D. Green, ${ }^{144}$ S. Grünendahl, ${ }^{144}$ O. Gutsche, ${ }^{144}$ R. M. Harris, ${ }^{144}$ S. Hasegawa, ${ }^{144}$ J. Hirschauer, ${ }^{144}$ Z. Hu, ${ }^{144}$ B. Jayatilaka, ${ }^{144}$ S. Jindariani, ${ }^{144}$ M. Johnson, ${ }^{144}$ U. Joshi, ${ }^{144}$ B. Klima, ${ }^{144}$ B. Kreis, ${ }^{144}$ S. Lammel, ${ }^{144}$ D. Lincoln, ${ }^{144}$ R. Lipton, ${ }^{144}$ M. Liu, ${ }^{144}$ T. Liu, ${ }^{144}$ R. Lopes De Sá ${ }^{144}$ J. Lykken, ${ }^{144}$ K. Maeshima, ${ }^{144}$ N. Magini, ${ }^{144}$ J. M. Marraffino, ${ }^{144}$ D. Mason, ${ }^{144}$ P. McBride,${ }^{144}$ P. Merkel, ${ }^{144}$ S. Mrenna, ${ }^{144}$ S. Nahn, ${ }^{144}$ V. O’Dell, ${ }^{144}$ K. Pedro, ${ }^{144}$ O. Prokofyev, ${ }^{144}$ G. Rakness, ${ }^{144}$ L. Ristori, ${ }^{144}$ B. Schneider, ${ }^{144}$ E. Sexton-Kennedy, ${ }^{144}$ A. Soha, ${ }^{144}$ W. J. Spalding, ${ }^{144}$ L. Spiegel, ${ }^{144}$ S. Stoynev, ${ }^{144}$ J. Strait, ${ }^{144}$ N. Strobbe,${ }^{144}$ L. Taylor,${ }^{144}$ S. Tkaczyk, ${ }^{144}$ N. V. Tran, ${ }^{144}$ L. Uplegger, ${ }^{144}$ E. W. Vaandering, ${ }^{144}$ C. Vernieri, ${ }^{144}$ M. Verzocchi, ${ }^{144}$ R. Vidal,,${ }^{144}$ M. Wang, ${ }^{144}$ H. A. Weber, ${ }^{144}$ A. Whitbeck, ${ }^{144}$ D. Acosta, ${ }^{145}$ P. Avery, ${ }^{145}$ P. Bortignon, ${ }^{145}$ D. Bourilkov, ${ }^{145}$ A. Brinkerhoff, ${ }^{145}$ A. Carnes, ${ }^{145}$ M. Carver, ${ }^{145}$ D. Curry, ${ }^{145}$ R. D. Field, ${ }^{145}$ I. K. Furic, ${ }^{145}$ S. V. Gleyzer, ${ }^{145}$ B. M. Joshi, ${ }^{145}$ J. Konigsberg, ${ }^{145}$ A. Korytov, ${ }^{145}$ K. Kotov, ${ }^{145}$ P. Ma ${ }^{145}$ K. Matchev, ${ }^{145}$ H. Mei, ${ }^{145}$ G. Mitselmakher, ${ }^{145}$ D. Rank, ${ }^{145}$ K. Shi, ${ }^{145}$ D. Sperka, ${ }^{145}$ N. Terentyev, ${ }^{145}$ L. Thomas, ${ }^{145}$

J. Wang, ${ }^{145}$ S. Wang, ${ }^{145}$ J. Yelton, ${ }^{145}$ Y. R. Joshi, ${ }^{146}$ S. Linn, ${ }^{146}$ P. Markowitz, ${ }^{146}$ J. L. Rodriguez, ${ }^{146}$ A. Ackert, ${ }^{147}$ T. Adams, ${ }^{147}$ A. Askew, ${ }^{147}$ S. Hagopian, ${ }^{147}$ V. Hagopian, ${ }^{147}$ K. F. Johnson, ${ }^{147}$ T. Kolberg, ${ }^{147}$ G. Martinez,${ }^{147}$ T. Perry, ${ }^{147}$ H. Prosper, ${ }^{147}$ A. Saha, ${ }^{147}$ A. Santra, ${ }^{147}$ V. Sharma, ${ }^{147}$ R. Yohay, ${ }^{147}$ M. M. Baarmand, ${ }^{148}$ V. Bhopatkar, ${ }^{148}$

S. Colafranceschi, ${ }^{148}$ M. Hohlmann, ${ }^{148}$ D. Noonan, ${ }^{148}$ T. Roy, ${ }^{148}$ F. Yumiceva, ${ }^{148}$ M. R. Adams, ${ }^{149}$ L. Apanasevich, ${ }^{149}$ D. Berry, ${ }^{149}$ R. R. Betts, ${ }^{149}$ R. Cavanaugh, ${ }^{149}$ X. Chen, ${ }^{149}$ O. Evdokimov, ${ }^{149}$ C. E. Gerber, ${ }^{149}$ D. A. Hangal, ${ }^{149}$ D. J. Hofman, ${ }^{149}$ K. Jung, ${ }^{149}$ J. Kamin, ${ }^{149}$ I. D. Sandoval Gonzalez, ${ }^{149}$ M. B. Tonjes ${ }^{149}$ H. Trauger, ${ }^{149}$ N. Varelas, ${ }^{149}$ H. Wang, ${ }^{149}$ Z. Wu, ${ }^{149}$ J. Zhang, ${ }^{149}$ B. Bilki, ${ }^{150, n n n}$ W. Clarida, ${ }^{150}$ K. Dilsiz, ${ }^{150, \text { ooo }}$ S. Durgut,${ }^{150}$ R. P. Gandrajula, ${ }^{150}$ M. Haytmyradov, ${ }^{150}$ V. Khristenko, ${ }^{150}$ J.-P. Merlo, ${ }^{150}$ H. Mermerkaya, ${ }^{150, p p p}$ A. Mestvirishvili, ${ }^{150}$ A. Moeller, ${ }^{150}$ J. Nachtman, ${ }^{150}$ H. Ogul, ${ }^{150, q q 9}$ Y. Onel, ${ }^{150}$ F. Ozok, ${ }^{150, r r}$ A. Penzo,${ }^{150}$ C. Snyder,${ }^{150}$ E. Tiras, ${ }^{150}$ J. Wetzel,${ }^{150}$ K. Yi, ${ }^{150}$

B. Blumenfeld, ${ }^{151}$ A. Cocoros, ${ }^{151}$ N. Eminizer, ${ }^{151}$ D. Fehling, ${ }^{151}$ L. Feng, ${ }^{151}$ A. V. Gritsan, ${ }^{151}$ P. Maksimovic, ${ }^{151}$ C. Mantilla, ${ }^{151}$ J. Roskes, ${ }^{151}$ U. Sarica ${ }^{151}$ M. Swartz,${ }^{151}$ M. Xiao, ${ }^{151}$ C. You, ${ }^{151}$ A. Al-bataineh,${ }^{152}$ P. Baringer, ${ }^{152}$ A. Bean, ${ }^{152}$ S. Boren, ${ }^{152}$ J. Bowen, ${ }^{152}$ J. Castle, ${ }^{152}$ S. Khalil, ${ }^{152}$ A. Kropivnitskaya, ${ }^{152}$ D. Majumder, ${ }^{152}$ W. Mcbrayer,${ }^{152}$ M. Murray, ${ }^{152}$ C. Royon, ${ }^{152}$ S. Sanders, ${ }^{152}$ E. Schmitz, ${ }^{152}$ J. D. Tapia Takaki, ${ }^{152}$ Q. Wang, ${ }^{152}$ A. Ivanov,${ }^{153}$ K. Kaadze,${ }^{153}$ Y. Maravin, ${ }^{153}$ A. Mohammadi, ${ }^{153}$ L. K. Saini, ${ }^{153}$ N. Skhirtladze, ${ }^{153}$ S. Toda,${ }^{153}$ F. Rebassoo, ${ }^{154}$ D. Wright, ${ }^{154}$ C. Anelli, ${ }^{155}$ A. Baden, ${ }^{155}$ O. Baron, ${ }^{155}$ A. Belloni, ${ }^{155}$ B. Calvert, ${ }^{155}$ S. C. Eno, ${ }^{155}$ Y. Feng,${ }^{155}$ C. Ferraioli, ${ }^{155}$ N. J. Hadley, ${ }^{155}$ S. Jabeen, ${ }^{155}$ G. Y. Jeng, ${ }^{155}$ 
R. G. Kellogg, ${ }^{155}$ J. Kunkle, ${ }^{155}$ A. C. Mignerey, ${ }^{155}$ F. Ricci-Tam, ${ }^{155}$ Y. H. Shin, ${ }^{155}$ A. Skuja, ${ }^{155}$ S. C. Tonwar, ${ }^{155}$ D. Abercrombie, ${ }^{156}$ B. Allen, ${ }^{156}$ V. Azzolini, ${ }^{156}$ R. Barbieri, ${ }^{156}$ A. Baty, ${ }^{156} \mathrm{R}$. Bi,${ }^{156}$ S. Brandt, ${ }^{156}$ W. Busza, ${ }^{156}$ I. A. Cali, ${ }^{156}$ M. D’Alfonso, ${ }^{156}$ Z. Demiragli, ${ }^{156}$ G. Gomez Ceballos, ${ }^{156}$ M. Goncharov, ${ }^{156}$ D. Hsu, ${ }^{156}$ M. Hu, ${ }^{156}$ Y. Iiyama,${ }^{156}$ G. M. Innocenti, ${ }^{156}$ M. Klute, ${ }^{156}$ D. Kovalskyi, ${ }^{156}$ Y. S. Lai, ${ }^{156}$ Y.-J. Lee, ${ }^{156}$ A. Levin, ${ }^{156}$ P. D. Luckey, ${ }^{156}$ B. Maier, ${ }^{156}$ A. C. Marini, ${ }^{156}$ C. Mcginn, ${ }^{156}$ C. Mironov, ${ }^{156}$ S. Narayanan, ${ }^{156}$ X. Niu, ${ }^{156}$ C. Paus, ${ }^{156}$ C. Roland, ${ }^{156}$ G. Roland, ${ }^{156}$ J. Salfeld-Nebgen, ${ }^{156}$ G. S. F. Stephans,${ }^{156}$ K. Tatar, ${ }^{156}$ D. Velicanu, ${ }^{156}$ J. Wang, ${ }^{156}$ T. W. Wang, ${ }^{156}$ B. Wyslouch, ${ }^{156}$ A. C. Benvenuti, ${ }^{157}$ R. M. Chatterjee, ${ }^{157}$ A. Evans, ${ }^{157}$ P. Hansen, ${ }^{157}$ J. Hiltbrand,${ }^{157}$ S. Kalafut,${ }^{157}$ Y. Kubota, ${ }^{157}$ Z. Lesko, ${ }^{157}$ J. Mans, ${ }^{157}$ S. Nourbakhsh, ${ }^{157}$ N. Ruckstuhl,${ }^{157}$ R. Rusack, ${ }^{157}$ J. Turkewitz, ${ }^{157}$ M. A. Wadud, ${ }^{157}$ J. G. Acosta, ${ }^{158}$ S. Oliveros, ${ }^{158}$ E. Avdeeva, ${ }^{159}$ K. Bloom, ${ }^{159}$ D. R. Claes, ${ }^{159}$ C. Fangmeier, ${ }^{159}$ R. Gonzalez Suarez, ${ }^{159}$ R. Kamalieddin, ${ }^{159}$ I. Kravchenko, ${ }^{159}$ J. Monroy, ${ }^{159}$ J. E. Siado, ${ }^{159}$ G. R. Snow, ${ }^{159}$ B. Stieger, ${ }^{159}$ J. Dolen, ${ }^{160}$ A. Godshalk, ${ }^{160}$ C. Harrington, ${ }^{160}$ I. Iashvili, ${ }^{160}$ D. Nguyen, ${ }^{160}$ A. Parker, ${ }^{160}$ S. Rappoccio, ${ }^{160}$ B. Roozbahani, ${ }^{160}$ G. Alverson, ${ }^{161}$ E. Barberis, ${ }^{161}$

A. Hortiangtham ${ }^{161}$ A. Massironi, ${ }^{161}$ D. M. Morse,${ }^{161}$ T. Orimoto, ${ }^{161}$ R. Teixeira De Lima ${ }^{161}$ D. Trocino, ${ }^{161}$ D. Wood, ${ }^{161}$ S. Bhattacharya, ${ }^{162}$ O. Charaf, ${ }^{162}$ K. A. Hahn, ${ }^{162}$ N. Mucia ${ }^{162}$ N. Odell, ${ }^{162}$ B. Pollack, ${ }^{162}$ M. H. Schmitt, ${ }^{162}$ K. Sung, ${ }^{162}$ M. Trovato, ${ }^{162}$ M. Velasco, ${ }^{162}$ N. Dev, ${ }^{163}$ M. Hildreth, ${ }^{163}$ K. Hurtado Anampa, ${ }^{163}$ C. Jessop, ${ }^{163}$ D. J. Karmgard, ${ }^{163}$ N. Kellams, ${ }^{163}$ K. Lannon, ${ }^{163}$ N. Loukas, ${ }^{163}$ N. Marinelli, ${ }^{163}$ F. Meng,${ }^{163}$ C. Mueller, ${ }^{163}$ Y. Musienko, ${ }^{163,11}$ M. Planer ${ }^{163}$ A. Reinsvold, ${ }^{163}$ R. Ruchti, ${ }^{163}$ G. Smith, ${ }^{163}$ S. Taroni, ${ }^{163}$ M. Wayne, ${ }^{163}$ M. Wolf, ${ }^{163}$ A. Woodard, ${ }^{163}$ J. Alimena, ${ }^{164}$ L. Antonelli, ${ }^{164}$ B. Bylsma, ${ }^{164}$ L. S. Durkin, ${ }^{164}$ S. Flowers, ${ }^{164}$ B. Francis, ${ }^{164}$ A. Hart, ${ }^{164}$ C. Hill, ${ }^{164}$ W. Ji, ${ }^{164}$ B. Liu, ${ }^{164}$ W. Luo, ${ }^{164}$ D. Puigh, ${ }^{164}$ B. L. Winer, ${ }^{164}$ H. W. Wulsin, ${ }^{164}$ S. Cooperstein, ${ }^{165}$ O. Driga, ${ }^{165}$ P. Elmer, ${ }^{165}$ J. Hardenbrook, ${ }^{165}$ P. Hebda, ${ }^{165}$ S. Higginbotham, ${ }^{165}$ D. Lange, ${ }^{165}$ J. Luo, ${ }^{165}$ D. Marlow, ${ }^{165}$ K. Mei, ${ }^{165}$ I. Ojalvo, ${ }^{165}$ J. Olsen, ${ }^{165}$ C. Palmer, ${ }^{165}$ P. Piroué, ${ }^{165}$ D. Stickland, ${ }^{165}$ C. Tully, ${ }^{165}$ S. Malik, ${ }^{166}$ S. Norberg, ${ }^{166}$ A. Barker,${ }^{167}$ V. E. Barnes, ${ }^{167}$ S. Das, ${ }^{167}$ S. Folgueras, ${ }^{167}$ L. Gutay, ${ }^{167}$ M. K. Jha, ${ }^{167}$ M. Jones ${ }^{167}$ A. W. Jung, ${ }^{167}$ A. Khatiwada ${ }^{167}$ D. H. Miller, ${ }^{167}$ N. Neumeister, ${ }^{167}$ C. C. Peng, ${ }^{167}$ H. Qiu, ${ }^{167}$ J. F. Schulte, ${ }^{167}$ J. Sun, ${ }^{167}$ F. Wang, ${ }^{167}$ W. Xie, ${ }^{167}$ T. Cheng, ${ }^{168}$ N. Parashar, ${ }^{168}$ J. Stupak, ${ }^{168}$ A. Adair, ${ }^{169}$ Z. Chen, ${ }^{169}$ K. M. Ecklund ${ }^{169}$ S. Freed, ${ }^{169}$ F. J. M. Geurts, ${ }^{169}$ M. Guilbaud, ${ }^{169}$ M. Kilpatrick, ${ }^{169}$ W. Li ${ }^{169}$ B. Michlin, ${ }^{169}$ M. Northup, ${ }^{169}$ B. P. Padley, ${ }^{169}$ J. Roberts, ${ }^{169}$ J. Rorie, ${ }^{169}$ W. Shi, ${ }^{169}$ Z. Tu, ${ }^{169}$ J. Zabel, ${ }^{169}$ A. Zhang, ${ }^{169}$ A. Bodek, ${ }^{170}$ P. de Barbaro, ${ }^{170}$ R. Demina, ${ }^{170}$ Y. t. Duh, ${ }^{170}$ T. Ferbel, ${ }^{170}$ M. Galanti, ${ }^{170}$ A. Garcia-Bellido, ${ }^{170}$ J. Han, ${ }^{170}$ O. Hindrichs, ${ }^{170}$

A. Khukhunaishvili, ${ }^{170}$ K. H. Lo, ${ }^{170}$ P. Tan, ${ }^{170}$ M. Verzetti, ${ }^{170}$ R. Ciesielski, ${ }^{171}$ K. Goulianos, ${ }^{171}$ C. Mesropian, ${ }^{171}$ A. Agapitos ${ }^{172}$ J. P. Chou, ${ }^{172}$ Y. Gershtein,${ }^{172}$ T. A. Gómez Espinosa, ${ }^{172}$ E. Halkiadakis, ${ }^{172}$ M. Heindl, ${ }^{172}$ E. Hughes,${ }^{172}$ S. Kaplan, ${ }^{172}$ R. Kunnawalkam Elayavalli, ${ }^{172}$ S. Kyriacou, ${ }^{172}$ A. Lath, ${ }^{172}$ R. Montalvo, ${ }^{172}$ K. Nash,${ }^{172}$ M. Osherson, ${ }^{172}$ H. Saka, ${ }^{172}$ S. Salur, ${ }^{172}$ S. Schnetzer, ${ }^{172}$ D. Sheffield, ${ }^{172}$ S. Somalwar, ${ }^{172}$ R. Stone, ${ }^{172}$ S. Thomas, ${ }^{172}$ P. Thomassen, ${ }^{172}$ M. Walker, ${ }^{172}$ A. G. Delannoy, ${ }^{173}$ M. Foerster, ${ }^{173}$ J. Heideman, ${ }^{173}$ G. Riley, ${ }^{173}$ K. Rose, ${ }^{173}$ S. Spanier, ${ }^{173}$ K. Thapa, ${ }^{173}$

O. Bouhali, ${ }^{174, \text { sss }}$ A. Castaneda Hernandez, ${ }^{174, \text { sss }}$ A. Celik, ${ }^{174}$ M. Dalchenko, ${ }^{174}$ M. De Mattia, ${ }^{174}$ A. Delgado, ${ }^{174}$

S. Dildick, ${ }^{174}$ R. Eusebi, ${ }^{174}$ J. Gilmore, ${ }^{174}$ T. Huang, ${ }^{174}$ T. Kamon, ${ }^{174, \text { ttt }}$ R. Mueller,${ }^{174}$ Y. Pakhotin, ${ }^{174}$ R. Patel, ${ }^{174}$ A. Perloff, ${ }^{174}$ L. Perniè, ${ }^{174}$ D. Rathjens, ${ }^{174}$ A. Safonov, ${ }^{174}$ A. Tatarinov, ${ }^{174}$ K. A. Ulmer, ${ }^{174}$ N. Akchurin, ${ }^{175}$ J. Damgov, ${ }^{175}$ F. De Guio, ${ }^{175}$ P. R. Dudero, ${ }^{175}$ J. Faulkner, ${ }^{175}$ E. Gurpinar, ${ }^{175}$ S. Kunori, ${ }^{175}$ K. Lamichhane, ${ }^{175}$ S. W. Lee, ${ }^{175}$ T. Libeiro, ${ }^{175}$

T. Peltola, ${ }^{175}$ S. Undleeb, ${ }^{175}$ I. Volobouev, ${ }^{175}$ Z. Wang, ${ }^{175}$ S. Greene, ${ }^{176}$ A. Gurrola, ${ }^{176}$ R. Janjam, ${ }^{176}$ W. Johns, ${ }^{176}$ C. Maguire, ${ }^{176}$ A. Melo, ${ }^{176}$ H. Ni, ${ }^{176}$ K. Padeken, ${ }^{176}$ P. Sheldon, ${ }^{176}$ S. Tuo, ${ }^{176}$ J. Velkovska, ${ }^{176}$ Q. Xu, ${ }^{176}$ M. W. Arenton, ${ }^{177}$ P. Barria, ${ }^{177}$ B. Cox,${ }^{177}$ R. Hirosky, ${ }^{177}$ M. Joyce, ${ }^{177}$ A. Ledovskoy,${ }^{177}$ H. Li,${ }^{177}$ C. Neu, ${ }^{177}$ T. Sinthuprasith, ${ }^{177}$ Y. Wang, ${ }^{177}$ E. Wolfe, ${ }^{177}$ F. Xia, ${ }^{177}$ R. Harr, ${ }^{178}$ P. E. Karchin, ${ }^{178}$ N. Poudyal,${ }^{178}$ J. Sturdy ${ }^{178}$ P. Thapa,${ }^{178}$ S. Zaleski, ${ }^{178}$ M. Brodski, ${ }^{179}$ J. Buchanan, ${ }^{179}$ C. Caillol, ${ }^{179}$ S. Dasu, ${ }^{179}$ L. Dodd, ${ }^{179}$ S. Duric, ${ }^{179}$ B. Gomber, ${ }^{179}$ M. Grothe, ${ }^{179}$ M. Herndon, ${ }^{179}$ A. Hervé, ${ }^{179}$ U. Hussain, ${ }^{179}$ P. Klabbers, ${ }^{179}$ A. Lanaro, ${ }^{179}$ A. Levine, ${ }^{179}$ K. Long, ${ }^{179}$ R. Loveless, ${ }^{179}$ G. Polese, ${ }^{179}$ T. Ruggles,${ }^{179}$ A. Savin, ${ }^{179}$ N. Smith, ${ }^{179}$ W. H. Smith, ${ }^{179}$ D. Taylor, ${ }^{179}$ and N. Woods ${ }^{179}$

(CMS Collaboration)

${ }^{1}$ Yerevan Physics Institute, Yerevan, Armenia

${ }^{2}$ Institut für Hochenergiephysik, Wien, Austria

${ }^{3}$ Institute for Nuclear Problems, Minsk, Belarus

${ }^{4}$ Universiteit Antwerpen, Antwerpen, Belgium

${ }^{5}$ Vrije Universiteit Brussel, Brussel, Belgium 


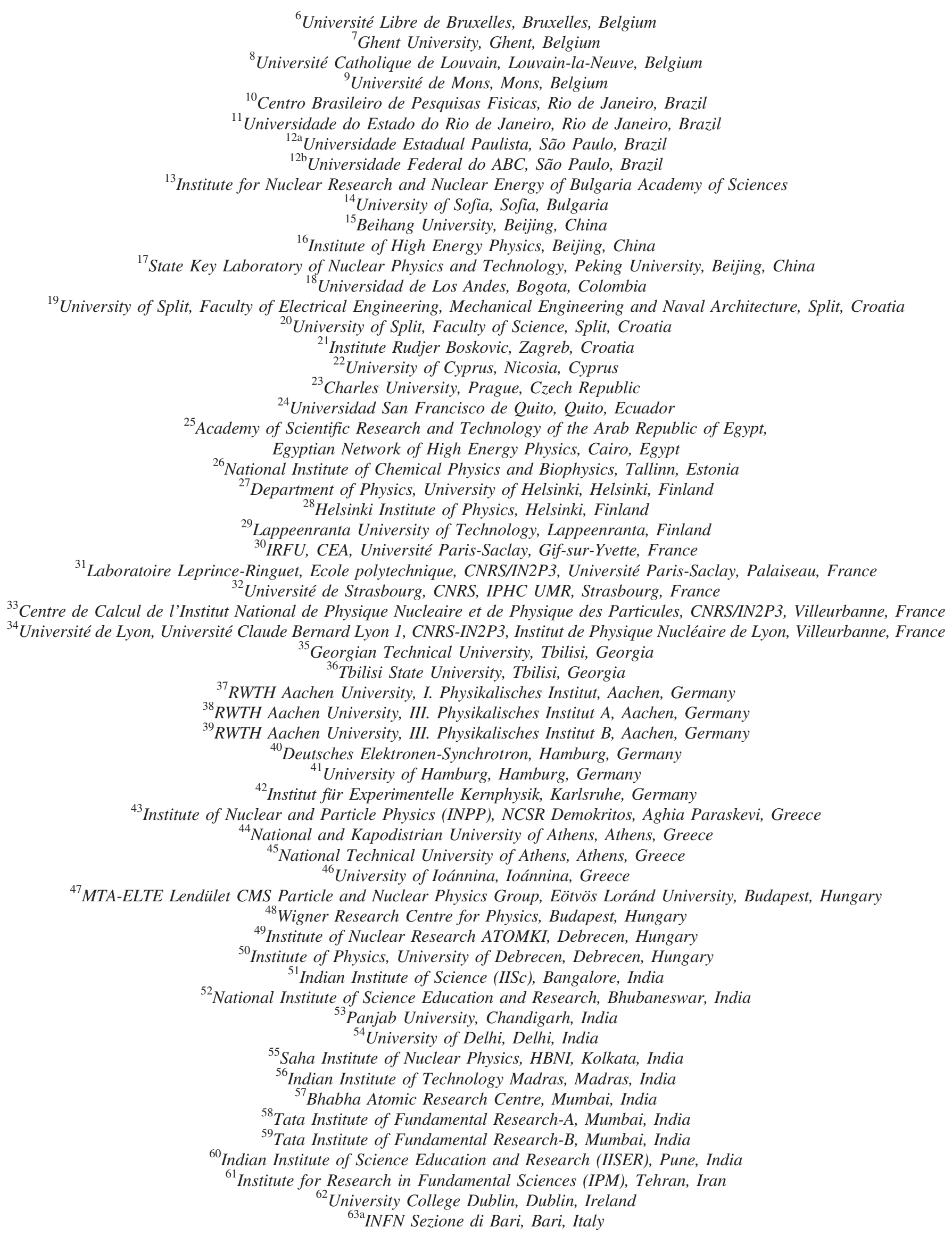




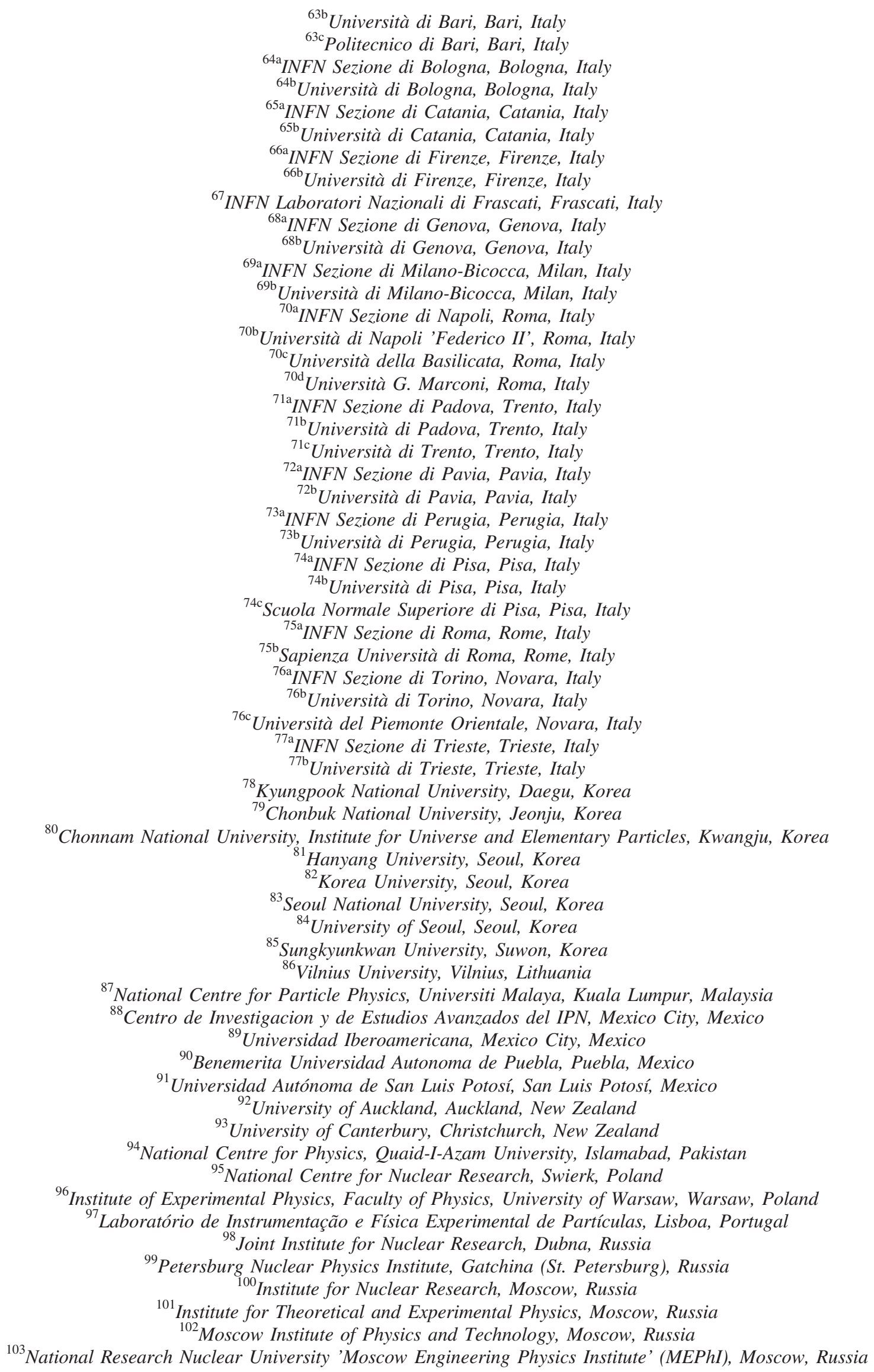


${ }^{104}$ P. N. Lebedev Physical Institute, Moscow, Russia

${ }^{105}$ Skobeltsyn Institute of Nuclear Physics, Lomonosov Moscow State University, Moscow, Russia

${ }^{106}$ Novosibirsk State University (NSU), Novosibirsk, Russia

${ }^{107}$ State Research Center of Russian Federation, Institute for High Energy Physics, Protvino, Russia

${ }^{108}$ University of Belgrade, Faculty of Physics and Vinca Institute of Nuclear Sciences, Belgrade, Serbia

${ }^{109}$ Centro de Investigaciones Energéticas Medioambientales y Tecnológicas (CIEMAT), Madrid, Spain

${ }^{110}$ Universidad Autónoma de Madrid, Madrid, Spain

${ }^{111}$ Universidad de Oviedo, Oviedo, Spain

${ }^{112}$ Instituto de Física de Cantabria (IFCA), CSIC-Universidad de Cantabria, Santander, Spain

${ }^{113}$ CERN, European Organization for Nuclear Research, Geneva, Switzerland

${ }^{114}$ Paul Scherrer Institut, Villigen, Switzerland

${ }^{115}$ Institute for Particle Physics and Astrophysics (IPA), Zurich, Switzerland

${ }^{116}$ Universität Zürich, Zurich, Switzerland

${ }^{117}$ National Central University, Chung-Li, Taiwan

${ }^{118}$ National Taiwan University (NTU), Taipei, Taiwan

${ }^{119}$ Chulalongkorn University, Faculty of Science, Department of Physics, Bangkok, Thailand

${ }^{120}$ Cukurova University, Physics Department, Science and Art Faculty, Adana, Turkey

${ }^{121}$ Middle East Technical University, Physics Department, Ankara, Turkey

${ }^{122}$ Bogazici University, Istanbul, Turkey

${ }^{123}$ Istanbul Technical University, Istanbul, Turkey

${ }^{124}$ Institute for Scintillation Materials of National Academy of Science of Ukraine, Kharkov, Ukraine

${ }^{125}$ National Scientific Center, Kharkov Institute of Physics and Technology, Kharkov, Ukraine

${ }^{126}$ University of Bristol, Bristol, United Kingdom

${ }^{127}$ Rutherford Appleton Laboratory, Didcot, United Kingdom

${ }^{128}$ Imperial College, London, United Kingdom

${ }^{129}$ Brunel University, Uxbridge, United Kingdom

${ }^{130}$ Baylor University, Waco, USA

${ }^{131}$ Catholic University of America, Washington DC, USA

${ }^{132}$ The University of Alabama, Tuscaloosa, USA

${ }^{133}$ Boston University, Boston, USA

${ }^{134}$ Brown University, Providence, USA

${ }^{135}$ University of California, Davis, Davis, USA

${ }^{136}$ University of California, Los Angeles, USA

${ }^{137}$ University of California, Riverside, Riverside, USA

${ }^{138}$ University of California, San Diego, La Jolla, USA

${ }^{139}$ University of California, Santa Barbara, Department of Physics, Santa Barbara, USA

${ }^{140}$ California Institute of Technology, Pasadena, USA

${ }^{141}$ Carnegie Mellon University, Pittsburgh, USA

${ }^{142}$ University of Colorado Boulder, Boulder, USA

${ }^{143}$ Cornell University, Ithaca, USA

${ }^{144}$ Fermi National Accelerator Laboratory, Batavia, USA

${ }^{145}$ University of Florida, Gainesville, USA

${ }^{146}$ Florida International University, Miami, USA

${ }^{147}$ Florida State University, Tallahassee, USA

${ }^{148}$ Florida Institute of Technology, Melbourne, USA

${ }^{149}$ University of Illinois at Chicago (UIC), Chicago, USA

${ }^{150}$ The University of Iowa, Iowa City, USA

${ }^{151}$ Johns Hopkins University, Baltimore, USA

${ }^{152}$ The University of Kansas, Lawrence, USA

${ }^{153}$ Kansas State University, Manhattan, USA

${ }^{154}$ Lawrence Livermore National Laboratory, Livermore, USA

${ }^{155}$ University of Maryland, College Park, USA

${ }^{156}$ Massachusetts Institute of Technology, Cambridge, USA

${ }^{157}$ University of Minnesota, Minneapolis, USA

${ }^{158}$ University of Mississippi, Oxford, USA

${ }^{159}$ University of Nebraska-Lincoln, Lincoln, USA

${ }^{160}$ State University of New York at Buffalo, Buffalo, USA

${ }^{161}$ Northeastern University, Boston, USA

${ }^{162}$ Northwestern University, Evanston, USA

${ }^{163}$ University of Notre Dame, Notre Dame, USA 


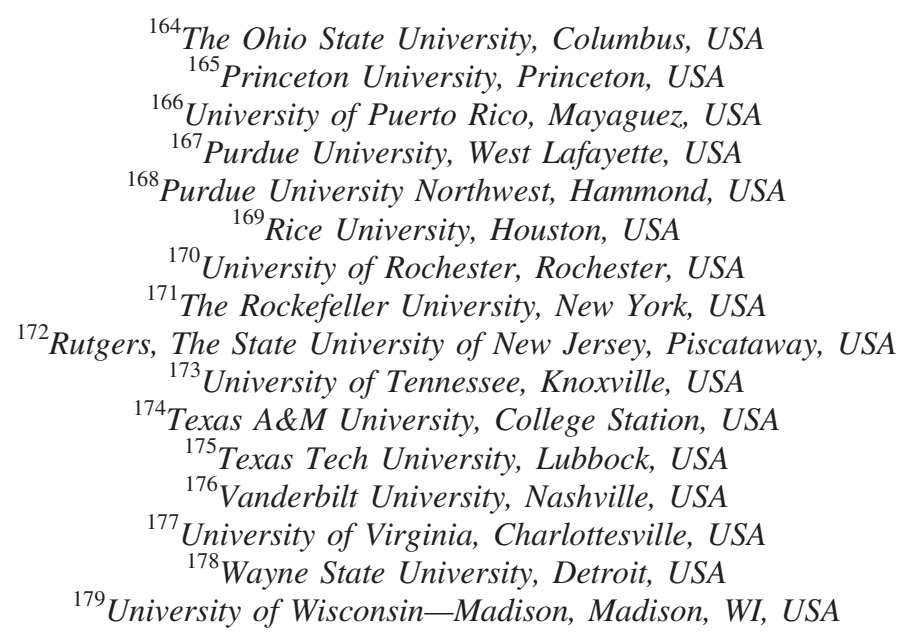

${ }^{\mathrm{a}}$ Deceased.

${ }^{\mathrm{b}}$ Also at Vienna University of Technology, Vienna, Austria.

${ }^{c}$ Also at State Key Laboratory of Nuclear Physics and Technology, Peking University, Beijing, China.

${ }^{\mathrm{d}}$ Also at IRFU, CEA, Université Paris-Saclay, Gif-sur-Yvette, France.

${ }^{\mathrm{e}}$ Also at Universidade Estadual de Campinas, Campinas, Brazil.

${ }^{\mathrm{f}}$ Also at Universidade Federal de Pelotas, Pelotas, Brazil.

${ }^{\mathrm{g}}$ Also at Université Libre de Bruxelles, Bruxelles, Belgium.

${ }^{\mathrm{h}}$ Also at Institute for Theoretical and Experimental Physics, Moscow, Russia.

${ }^{\mathrm{i}}$ Also at Joint Institute for Nuclear Research, Dubna, Russia.

${ }^{\mathrm{j} A l s o}$ at Suez University, Suez, Egypt.

${ }^{\mathrm{k}}$ Also at British University in Egypt, Cairo, Egypt.

${ }^{1}$ Also at Fayoum University, El-Fayoum, Egypt.

${ }^{\mathrm{m}}$ Also at Helwan University, Cairo, Egypt.

${ }^{\mathrm{n}}$ Also at Université de Haute Alsace, Mulhouse, France.

${ }^{\circ}$ Also at Skobeltsyn Institute of Nuclear Physics, Lomonosov Moscow State University, Moscow, Russia.

${ }^{\mathrm{p}}$ Also at CERN, European Organization for Nuclear Research, Geneva, Switzerland.

${ }^{\mathrm{q}}$ Also at RWTH Aachen University, III. Physikalisches Institut A, Aachen, Germany.

${ }^{\mathrm{r}}$ Also at University of Hamburg, Hamburg, Germany.

${ }^{\text {s}}$ Also at Brandenburg University of Technology, Cottbus, Germany.

${ }^{t}$ Also at MTA-ELTE Lendület CMS Particle and Nuclear Physics Group, Eötvös Loránd University, Budapest, Hungary.

uAlso at Institute of Nuclear Research ATOMKI, Debrecen, Hungary.

${ }^{v}$ Also at Institute of Physics, University of Debrecen, Debrecen, Hungary.

${ }^{\mathrm{w}}$ Also at IIT Bhubaneswar, Bhubaneswar, India.

${ }^{\mathrm{x}}$ Also at Institute of Physics, Bhubaneswar, India.

${ }^{y}$ Also at University of Visva-Bharati, Santiniketan, India.

${ }^{\mathrm{z}}$ Also at University of Ruhuna, Matara, Sri Lanka.

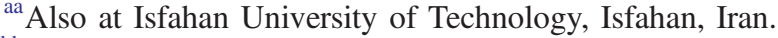

${ }^{\mathrm{bb}}$ Also at Yazd University, Yazd, Iran.

${ }^{c c}$ Also at Plasma Physics Research Center, Science and Research Branch, Islamic Azad University, Tehran, Iran.

${ }^{\mathrm{dd}}$ Also at Università degli Studi di Siena, Siena, Italy

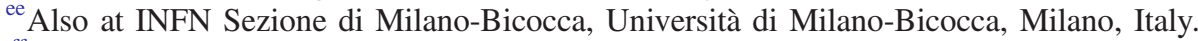

${ }^{\mathrm{ff}}$ Also at Laboratori Nazionali di Legnaro dell'INFN, Legnaro, Italy.

${ }^{g g}$ Also at Purdue University, West Lafayette, USA.

${ }^{\mathrm{hh}}$ Also at International Islamic University of Malaysia, Kuala Lumpur, Malaysia.

${ }^{\text {ii } A l s o ~ a t ~ M a l a y s i a n ~ N u c l e a r ~ A g e n c y, ~ M O S T I, ~ K a j a n g, ~ M a l a y s i a . ~}$

${ }^{\mathrm{jj}}$ Also at Consejo Nacional de Ciencia y Tecnología, Mexico City, Mexico.

${ }^{\mathrm{kk}}$ Also at Warsaw University of Technology, Institute of Electronic Systems, Warsaw, Poland.

${ }^{11}$ Also at Institute for Nuclear Research, Moscow, Russia.

${ }^{\mathrm{mm}}$ Also at National Research Nuclear University 'Moscow Engineering Physics Institute' (MEPhI), Moscow, Russia.

${ }^{\mathrm{nn}}$ Also at St. Petersburg State Polytechnical University, St. Petersburg, Russia.

${ }^{\mathrm{oo}}$ Also at University of Florida, Gainesville, USA.

${ }^{\mathrm{pp}}$ Also at P. N. Lebedev Physical Institute, Moscow, Russia.

${ }^{\mathrm{qq}}$ Also at California Institute of Technology, Pasadena, USA. 


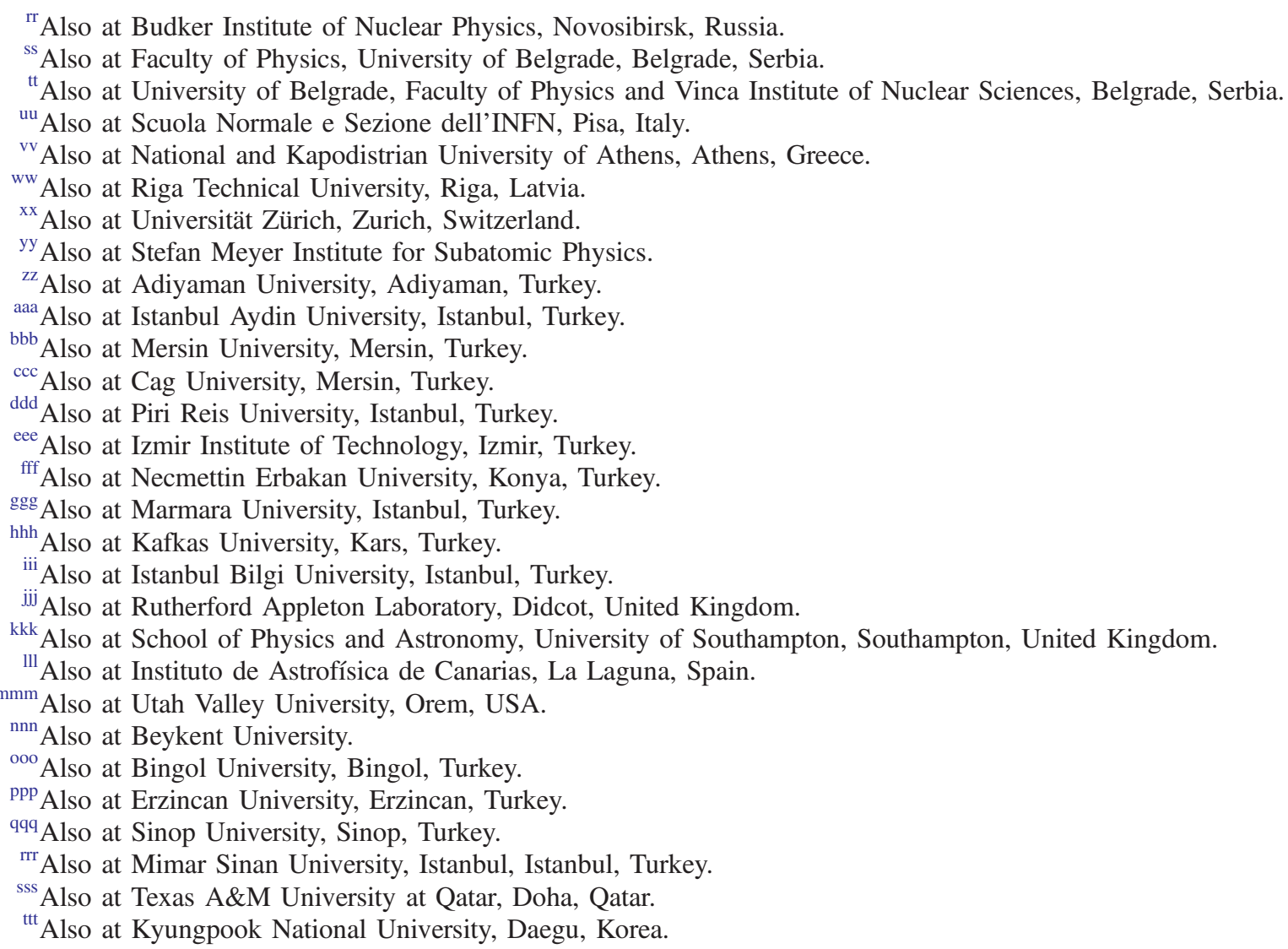

